\title{
sRNAs enriched in outer membrane vesicles of pathogenic Flavobacterium psychrophilum interact with immune genes of rainbow trout
}

5

6

\section{Abstract}

10 Outer membrane vesicles (OMVs) released by gram-negative bacteria during host-pathogen

11 interactions harbor cargos, such as DNA, RNA, toxins, and virulence factors. We hypothesized

12 that sRNAs carried within OMVs of Flavobacterium psychrophilum interact with host immune

13 genes and affect their expression. OMVs were isolated from F. psychrophilum and visualized

14 using transmission electron microscopy (TEM). RNA-Seq datasets generated from whole-cell $F$.

15 psychrophilum and their OMVs indicated enrichment of specific sRNAs in the OMVs compared

16 to the parent cell. Fluorescent in situ hybridization (FISH) and confocal microscopy confirmed

17 the expression of a randomly chosen sRNA.

18 Integrated RNA-Seq analyses of host transcriptome and bacterial sRNAs on day 5 post-

19 infection of $F$. psychrophilum-resistant and -susceptible rainbow trout genetic lines revealed 516

20 protein-coding, 595 lncRNA, and 116 bacterial sRNA differentially expressed (DE) transcripts.

21 Integrated and network analyses of these DE transcripts revealed immune genes targeted by

22 bacterial sRNAs. On the top of these genes, an isoform encoding anaphase-promoting complex

23 subunit 13 (ANAPC13_1) was highly upregulated and exhibited interaction and reciprocal

24 expression with 21 DE sRNAs enriched in OMVs and/or located in pathogenicity islands (PAIs).

25 In vitro treatment of the rainbow trout epithelial cell line RTgill-W1 with OMVs showed signs of

26 cell autolysis accompanied by dynamic changes in expression of host genes when profiled $24 \mathrm{~h}$ 
27 following treatment. The OMV-enriched sRNAs, soFE013584 and soFE002123, showed high

28 interactions with the protection of telomeres 1 gene (POT1); essential for chromosome stability

29 and cellular viability. Modulation of the host gene expression following OMV-treatment, which

30 favors elements from the phagocytic, endocytic, and antigen presentation pathways in addition to

31 HSP70, HSP90, and cochaperone proteins, provided evidence for a potential role of OMVs in

32 boosting the host immune response. In conclusion, our work identified novel microbial targets

33 and inherent characteristics of OMVs that could open up new avenues of treatment and prevention

34 of fish infections.

35 Keywords: Aquaculture, rainbow trout, Flavobacterium psychrophilum, OMVs, small noncoding 36 RNA (sRNA), bacterial cold water disease, disease resistance.

37 Corresponding author: Mohamed Salem, mosalem@umd.edu

38 The first two authors equally contributed to this work.

41 Introduction

42 Flavobacterium psychrophilum causes Bacterial cold-water disease (BCWD), a severe

43 infection that frequently affects salmonid species, including salmon and rainbow trout. BCWD is

44 responsible for causing a considerable economic loss in freshwater aquaculture of salmonids [1].

45 Losses from this infection have been reported in many countries, including the United States,

46 United Kingdom, Australia, and Japan [2]. Mortality rates from this disease vary, but rates as high

47 as $90 \%$ have been reported [3]. F. psychrophilum is a psychrophilic, gram-negative bacterium

48 typically found in cold freshwater [4]. After entering the host, likely through skin abrasions, $F$.

49 psychrophilum spreads through the dermis, connective, and muscle tissues [1]. Rainbow trout

50 (especially fry) infected by F. psychrophilum exhibit irregular swimming behavior, exophthalmia,

51 darkened coloration, skeletal deformities, and visible deep necrotic lesions around the caudal 
52 peduncle [3]. The survivability of this pathogen under various environmental conditions and the

53 unavailability of vaccines contribute to the severity of the disease [5].

54 Efforts have been made to create BCWD-resistant strains of rainbow trout. Recent approaches

55 have correlated fish genetic variations to immune responsiveness and survival rates after

56 challenges with $F$. psychrophilum [6-8]. Through a selective breeding program, the National

57 Center for Cool and Cold-Water Aquaculture (NCCCWA) developed three genetic lines of

58 rainbow trout: $\mathrm{BCWD}$-susceptible, -control, and -resistant lines [6, 9]. The susceptible line has a

59 very low survival rate $(29.4 \%)$, the control line has an intermediate survival rate $(54.6 \%)$, and the

60 resistant line has undergone multiple generations of selection against $F$. psychrophilum challenge

61 to enhance their survival rate up to $94 \%$ [6].

62 The molecular pathogenesis of F. psychrophilum is still not yet completely understood. Many

63 factors, including biofilm formation, secretion systems, and virulence factors such as exotoxins,

64 proteases, and adhesins, are thought to play a role in the pathogenesis of this bacterium [1, 10]. F.

65 psychrophilum, similar to other gram-negative bacteria, produce small $(<300 \mathrm{~nm}$ in diameter $)$

66 spherical particles derived from the bacterial outer membrane, commonly known as outer

67 membrane vesicles (OMVs) [11]. Many pathogenic bacteria rely on OMVs to deliver virulence

68 factors to their host $[12,13]$, and OMV-mediated delivery has been considered an essential

69 mechanism in host-pathogen interactions. For example, OMVs of Porphyromonas gingivalis

70 contain a group of proteases, constituting the virulence factor "gingipains", which degrade

71 cytokines and ultimately downregulate the immune response of host cells [14]. Similarly, different

72 cargos, such as DNA, RNA, and cytosolic proteins within OMVs, can be transported to the host

73 cell when OMVs fuse with host cell membranes or bind to the host cell receptors [15-17]. sRNAs 
74 within OMVs of Pseudomonas aeruginosa have been shown to reduce the host immune response $75[16]$.

76 Bacterial sRNAs are non-protein-coding molecules with a length typically ranging from 50 to

77500 nucleotides and have essential roles in post-transcriptional gene regulation [18, 19]. sRNAs

78 usually originate from the untranslated regions of the bacterial genome but can also be acquired

79 by horizontal gene transfer $[18,20]$. Many sRNAs act as complementary antisense sequences to

80 mRNAs [21], and their function resembles that of the eukaryotic microRNAs (miRNAs). They

81 cause translational repression by directly hybridizing with mRNAs to promote transcript

82 degradation [22]. These molecular mechanisms allow sRNAs to play a role in bacterial

83 pathogenicity. For example, a few studies have suggested that the network of bacterial genes in

84 biofilm formation is controlled by sRNAs [23, 24]. A recent study in $P$. aeruginosa has shown

85 the involvement of sRNAs in repressing the translation of genes involved in quorum sensing by

86 binding to the ribosome binding sites of mRNA targets [25]. A study on Staphylococcus aureus

87 reported the participation of sRNAs in regulating cellular responses to signal molecules [26].

88 Another study reported that entry of bacterial sRNAs inside host cells triggers a host gene-bacterial

89 sRNAs interaction resulting in degradation of host immune genes [27].

90 In this study, OMVs were isolated from F. psychrophilum (strain CSF-259-93) broth culture

91 on day 8 of bacterial growth. The parent bacteria and OMVs were subjected to RNA sequencing,

92 and then the transcriptomic data were used for sRNAs prediction. We identified sRNAs specific

93 and/or enriched in OMVs by comparing sRNA expression levels in the parent cells versus OMVs.

94 Further, in vivo investigated the interactions among bacterial sRNAs within F. psychrophilum

95 OMVs and rainbow trout immune genes. Dual RNA-Seq of host and pathogen revealed that

96 bacterial sRNAs were reciprocally expressed with their target immune genes on day 5 following 
97 infection of selectively bred resistant-, and susceptible-line rainbow trout. These results suggest a

98 role for bacterial sRNAs during host-pathogen interactions, facilitating bacterial invasion/growth.

99 We then in vitro investigated the effect of OMVs on RTgill-W1 host cells and the interaction

100 between bacterial sRNA and host genes. OMVs caused host cell lysis; however, immune response

101 genes expression was boosted at the early phase of OMV treatment (24h post-treatment).

\section{Results}

103 OMVs extraction and TEM

104 OMVs were isolated from bacterial cells for downstream RNA sequencing and prediction of

105 bacterial sRNAs. The experimental design is shown in Fig. 1. F. psychrophilum colonies grown

106 on Tryptone Yeast Extracts (TYEs) agar plates were observed as bright yellow colonies after 5

107 days of incubation at $15^{\circ} \mathrm{C}$. In broth culture, bacterial log phase growth was observed from day 5

108 until day 11 (Fig. 2a). Isolated F. psychrophilum OMVs were observed by transmission electron

109 microscopy (TEM) as spherical-shaped particles with an average diameter of 50-100 nm (Fig. 2b),

110 whereas F. psychrophilum was observed as having rod-shaped morphology with a size of

111 approximately 3-5 $\mu \mathrm{m}$ (Fig. 2c).

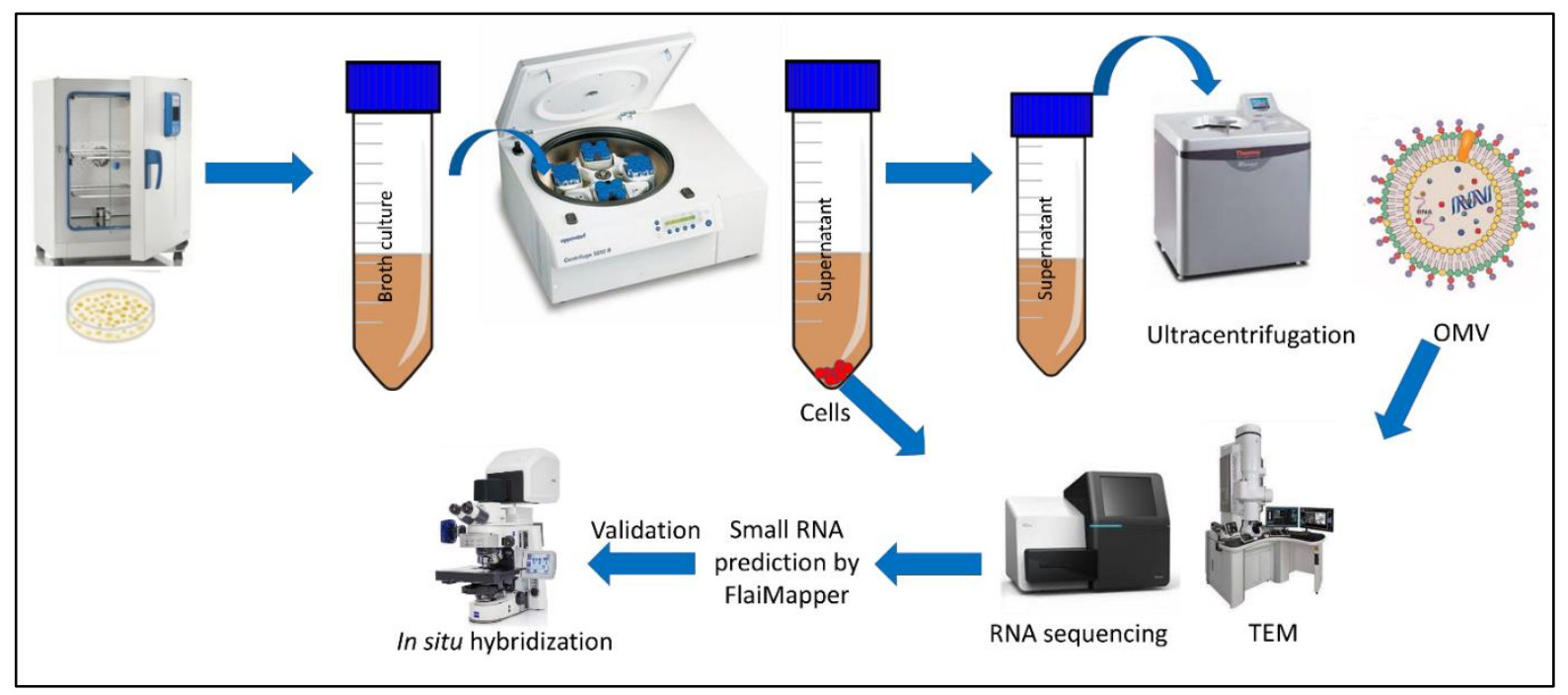


Fig. 1: Overview of the experimental design to predict and validate bacterial small RNAs. Frozen stock cultures of $F$. psychrophilum were cultured on TYEs agar, and the plate was incubated at $15^{\circ} \mathrm{C}$ for one week. F. psychrophilum colonies isolated from TYEs agar plate were transferred to TYEs broth. For OMV isolation, broth culture tubes were centrifuged to pellet the bacterial cells, and the supernatant was collected and filtered to remove any remaining bacterial cells. The filtrate was then subjected to ultracentrifugation to pellet the OMVs. F. psychrophilum cells and OMVs were visualized using TEM, followed by RNA sequencing on an Illumina MiSeq platform. Small RNAs were predicted using FlaiMapper, and validated by FISH.

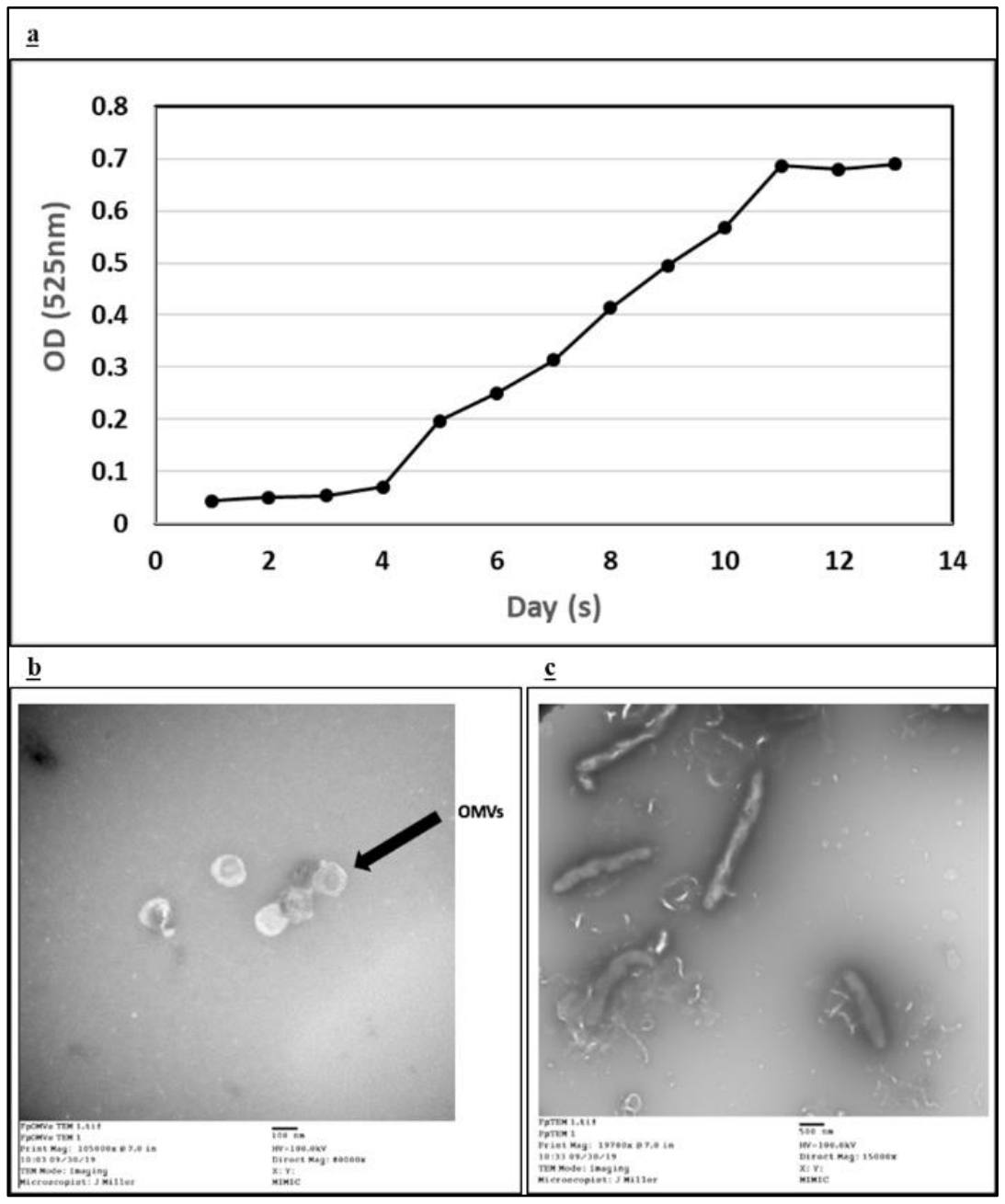

123 Fig. 2: a) Measurement of $F$. psychrophilum density by measuring OD in broth culture, the log124 phase was observed between days 5 and 11. b) Transmission Electron Microscopy (TEM) of $F$. 125 psychrophilum OMVs. OMVs appeared as spherical-shaped particles, and the black arrow points 126 to the bilayered OMVs' spherical structure. c) TEM of $F$. psychrophilum, which appeared as a 127 rod-shaped structure. 
RNA-Seq identifies sRNAs enriched in OMVs

130 Total RNA isolated from whole-cells and OMVs was sequenced, yielding 60,352,578 and

$13155,722,742$ sequence reads, respectively. High-quality reads from whole-cell and OMVs were

132 separately mapped to the F. psychrophilum reference genome, where $80.0 \%$ and $80.3 \%$ read

133 mapping rates were achieved, respectively. FlaiMapper [28] was used to predict small noncoding

134 RNAs from the alignment files. In total, 118,876 and 134,518 noncoding transcripts were

135 predicted from the whole-cells and OMVs, respectively. Transcripts longer than 500 bp were

136 filtered out, yielding 118,305 and 133,815 predicted sRNAs from the whole-cells and OMVs,

137 respectively. The sRNA length ranged from 87 to 499 nucleotides (Average $100.4 \mathrm{nt}$ ). The

138 majority of the predicted sRNAs $(98.9 \%)$ were $\leq 100 \mathrm{bp}$ in length. The list of predicted sRNAs

139 with their genomic location is included in Additional file 1 (Tables S1 \& S2). The sRNA

140 soFE128978 was randomly selected for validation and visualization by confocal microscopy. The

141 Cy3 fluorophore was used to visualize the sRNA. Fluorescent in situ hybridization and confocal

142 imaging confirmed the expression of the sRNA within F. psychrophilum (Fig. 3). The red color

143 in F. psychrophilum using an antisense probe and no fluorescence in F. psychrophilum using a

144 non-sense probe (negative control) validates the results.

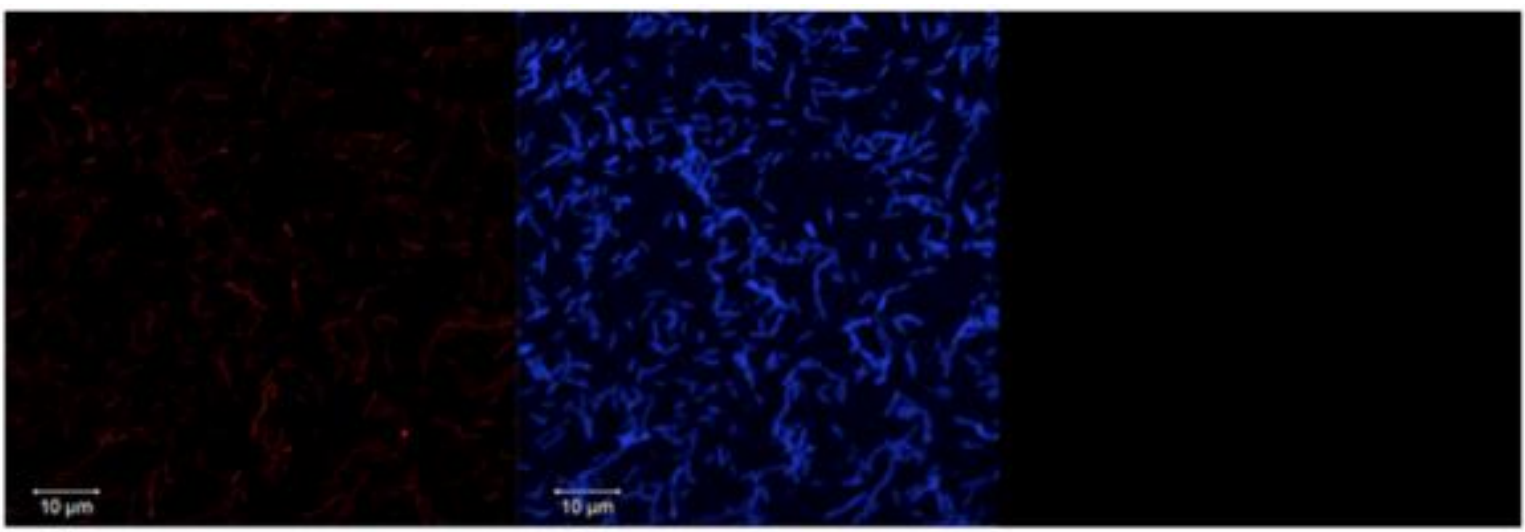

146 Fig. 3: Confocal micrograph of FISH stained F. psychrophilum cells using Cy3-labeled 147 SoFE128978 probe (Red) on the left panel. No fluorescence was observed in F. psychrophilum 
148 using a non-sense probe on the right panel (negative control) and $F$. psychrophilum cell stained by

149 DAPI in the center panel.

150

RNA-Seq of the whole-cell and OMVs revealed 11,015 and 10,437 sRNAs, respectively, with

152 Transcripts Per Million (TPM) expression level greater than 0.5. Of them, 5,975 sRNAs in OMVs

153 and 5,947 sRNAs in the whole cell had TPM >1. The expression value of sRNAs in OMVs and

154 the whole cell are included in Additional file 1 (Tables S1 \& S2). sRNAs were classified as OMV-

155 specific when OMV sRNAs share less than 90\% sequence overlap with sRNAs in the whole cell.

156 Otherwise, they were considered "common" sRNAs. We reported the sRNAs classification in

157 Additional file 1 (Tables S3 \& S4). In total, 108 OMV-specific sRNAs with TPM > 0.5 were

158 identified (Table 1 and Additional file 1; Table S3). Also, sRNAs were classified as OMV-

159 enriched if their TPM expression was at least two times higher than the whole cell $\left(\log _{2}\right.$ TPM ratio

$160 \geq 1$ ). $17.23 \%$ of the common sRNAs were OMV-enriched (Table 2 and Additional file 1; Table

161 S4). The OMV-specific or -enriched sRNAs could be explained by the selective inclusion of

162 sRNAs in the OMV, which suggests potential roles of these sRNAs in microbe-host interaction.

163 Consistent with our data, a previous study reported enrichment of sRNAs in $P$. aeruginosa OMVs

$164[16]$.

165 Table 1: A subset of OMV-specific sRNAs with TPM > 1.5; PAI denotes sRNAs located in 166 Pathogenicity Islands.

167

\begin{tabular}{|c|c|c|c|c|}
\hline sRNA & Start & End & TPM & PAl \\
\hline soFE129980 & 607022 & 607121 & 829.95 & Yes \\
\hline soFE081095 & 2583062 & 2583161 & 13.10 & No \\
\hline soFE023505 & 490867 & 490966 & 7.14 & No \\
\hline soFE113400 & 2080493 & 2080592 & 5.60 & No \\
\hline soFE014089 & 490862 & 490961 & 5.44 & No \\
\hline soFE024485 & 490852 & 490951 & 2.27 & No \\
\hline soFE068150 & 939025 & 939124 & 2.16 & No \\
\hline soFE038667 & 2656387 & 2656486 & 2.11 & No \\
\hline
\end{tabular}




\begin{tabular}{|c|c|c|c|c|}
\cline { 1 - 2 } SoFE132118 & 1665123 & 1665222 & 1.90 & Yes \\
\hline SoFE005824 & 1180568 & 1180667 & 1.86 & No \\
\hline SoFE017951 & 924082 & 924181 & 1.78 & No \\
\hline SoFE070737 & 209041 & 209140 & 1.58 & No \\
\hline SoFE071960 & 1201207 & 1201306 & 1.52 & No \\
\hline SoFE075699 & 1480007 & 1480106 & 1.52 & No \\
\hline
\end{tabular}

Table 2: A subset sRNAs exhibiting enriched expression in the OMVs compared to the wholecell. $\log _{2}$ ratio is the logarithmic value obtained by dividing the TPM expression of each sRNA in OMVs by that of the whole cell. PAI denotes sRNAs located in Pathogenicity Islands.

\begin{tabular}{|c|c|c|c|c|c|c|}
\hline SRNA & Start & End & TPM (OMV) & TPM (cell) & $\log _{2}$ ratio & PAI \\
\hline soFE000222 & 2080716 & 2080815 & 608.38 & 0.96 & 9.32 & No \\
\hline soFE000671 & 2080722 & 2080821 & 199.97 & 0.96 & 7.71 & No \\
\hline soFE000141 & 2080709 & 2080808 & 743.95 & 3.80 & 7.61 & No \\
\hline soFE001158 & 2080734 & 2080833 & 18.32 & 0.11 & 7.41 & No \\
\hline soFE000208 & 2080699 & 2080798 & 822.14 & 7.66 & 6.75 & No \\
\hline soFE000754 & 2080729 & 2080828 & 43.14 & 0.66 & 6.03 & No \\
\hline soFE017190 & 490892 & 490991 & 12.79 & 0.69 & 4.21 & No \\
\hline soFE017916 & 490900 & 490999 & 12.94 & 0.77 & 4.07 & No \\
\hline soFE031643 & 490880 & 490979 & 8.79 & 0.59 & 3.91 & No \\
\hline soFE000238 & 2580160 & 2580259 & 958.52 & 66.57 & 3.85 & No \\
\hline soFE000237 & 2092686 & 2092785 & 958.51 & 66.58 & 3.85 & No \\
\hline soFE000236 & 1662630 & 1662729 & 958.54 & 66.58 & 3.85 & Yes \\
\hline soFE005377 & 2046610 & 2046709 & 6.45 & 1.74 & 1.89 & No \\
\hline soFE036061 & 2305986 & 2306085 & 0.54 & 0.15 & 1.88 & No \\
\hline soFE007845 & 2046631 & 2046730 & 7.30 & 1.99 & 1.87 & No \\
\hline soFE010415 & 862382 & 862481 & 1.24 & 0.40 & 1.65 & No \\
\hline
\end{tabular}

175 Ten genomic islands were predicted in F. psychrophilum by at least one computational method

176 (Fig. 4). The full output table from the IslandViewer showing genomic island size, location, and 177 annotation is included in Additional file 2 (Table S5). 863 sRNAs with expression level TPM > 1

178 were predicted in the PAIs (Additional file 2; Table S6). For instance, the OMV-specific sRNA 179 soFE129980 was located within a PAI spanning the genomic region 583,837-607,648. Other 180 genes within this PAI included transposase, integrase, universal stress protein UspA, and 
181 transcription termination/anti-termination protein NusA (Fig. 4). Also, we identified an OMV-

182 specific sRNA, SoFE132118, spanning the genomic region 1,660,689-1,665,551. Due to their

183 pathogenic potential, we sought to investigate the interaction of OMV-specific sRNAs (Table 1)

184 with immune-related target genes. Marancik et al. [6] identified 2,633 putative immune relevant

185 genes by GO and manual annotations of the first draft of the trout genome [29]. We remapped

186 these sequences to a newer reference of the rainbow trout genome. Sequences were mapped to

1872,101 genomic loci. We selected the longest transcript generated from each locus to investigate

188 the OMV sRNA-host immune gene interactions. Fourteen sRNAs (Table 1) exhibited

189 computationally predicted interaction with $69.6 \%$ of the host immune transcripts (Additional file

190 2; Table S7). For instance, sRNA soFE129980 targeted 1,117 of the host immune-relevant genes;

191 of them, 151 genes exhibited very strong interactions (ndG cutoff -0.2). These results suggest a

192 potential role for the OMV-sRNAs in mediating host-pathogen interactions to facilitate pathogen

193 invasion/growth.

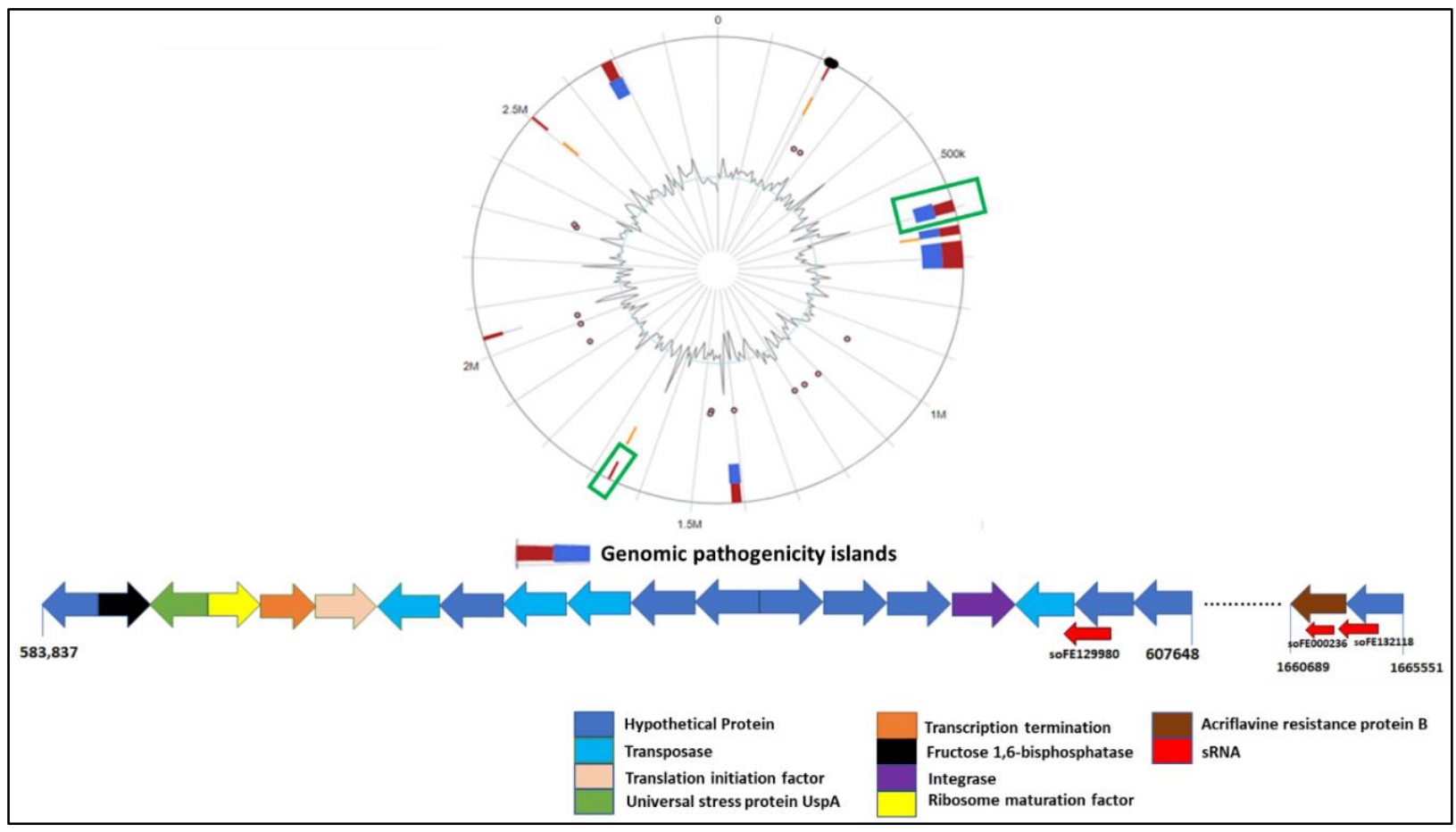


Fig. 4: Genomic pathogenicity islands (clusters of genes that play a role in microbial adaptability) of F. psychrophilum predicted by either IslandPath-DIMOB alone (Blue) or integrated methods (at least two methods, Red). The green windows indicate the location of 3

198 sRNAs, soFE129980, soFE132118 and soFE000236, within genomic pathogenicity islands. 199 Color-coded arrows, representing the physical location of genes within PAIs, are annotated at the 200 bottom of the figure, where the red arrows indicate sRNAs.

\section{Dual RNA-Seq of host and pathogen on day 5 post-infection of fish from resistant and} susceptible genetic lines

204 In this study, we aimed to investigate host-pathogen interactions, in vivo, on day 5 following

205 infection of rainbow trout with F. psychrophilum. In particular, we sought to investigate the

206 potential role of bacterial sRNAs in disease susceptibility and identify host immune-related genes

207 and lncRNAs that are likely targeted by these sRNAs. For this purpose, we used fish collected

208 from selectively bred resistant (ARS-Fp-R) and susceptible (ARS-Fp-S) genetic lines challenged

209 with $F$. psychrophilum as previously described in [6]. Total RNA was sequenced from 8 pooled

210 samples for host mRNAs, lncRNAs, and bacterial sRNAs (see Methods section). RNA-Seq from

211 infected resistant and susceptible genetic lines yielded a total of 372,100,715 raw sequence reads

212 (average of 46,512,589 reads/sample) with 12.1 depth of coverage. Sequence reads were

213 trimmed/filtered to generate 371,959,119 high-quality reads (average of 46,494,890 reads/sample).

214 To identify DE transcripts, high-quality reads were separately mapped to the reference genomes

215 of the host and pathogen (Fig. 5a). A total of 285,744,993 (76.8\%) trimmed reads were mapped

216 to the rainbow trout genome. In our previous studies, 59.9\% of the total RNA-sequence reads

$217(518,881,838)$ were mapped to the trout references [6, 7]. Additionally, 4,729,437 reads $(1.27 \%)$

218 were mapped to the F. psychrophilum reference genome. Notably, 99.4\% of the F. psychrophilum

219 mapped reads were generated from the susceptible genetic line. A higher bacterial load was

220 previously reported in the susceptible line when compared to the resistant line [6]. Normalized 
221 gene expression was used to account for differences across samples by converting raw count data

222 to Transcripts Per Kilobase Million (TPM) format. Quality and mapping statistics of sequence

223 reads are given in Additional file 3; Table S8. A total of 516 (483 loci) protein-coding, 595

224 IncRNA, and 116 bacterial sRNA transcripts were DE with FDR $<0.05$ and a minimum $\log _{2}$ fold

225 change value $\geq 1$ or $\leq-1$ (Fig. $5 b$ \& 5 c and Additional file 3; Tables S9, S10 \& S11). Of the DE

226 protein-coding transcripts, 82 had immune-related functions, as we previously reported [6]. Most

227 of the DE protein-coding (86.2\%) and lncRNA transcripts (68.7\%) were downregulated in the

228 resistant line. In our previous study, 54 out of 83 lncRNAs (65.1\%) were downregulated on day

2295 post-infection [7]. Notably, 26.5\% of the differentially regulated lncRNAs previously described

230 in [7] were also identified in the current study, suggesting the potential role of these genes in

231 resistance to BCWD. More information about DE transcripts is given in Additional file 3

232 (Tables S9, S10 \& S11). 
a

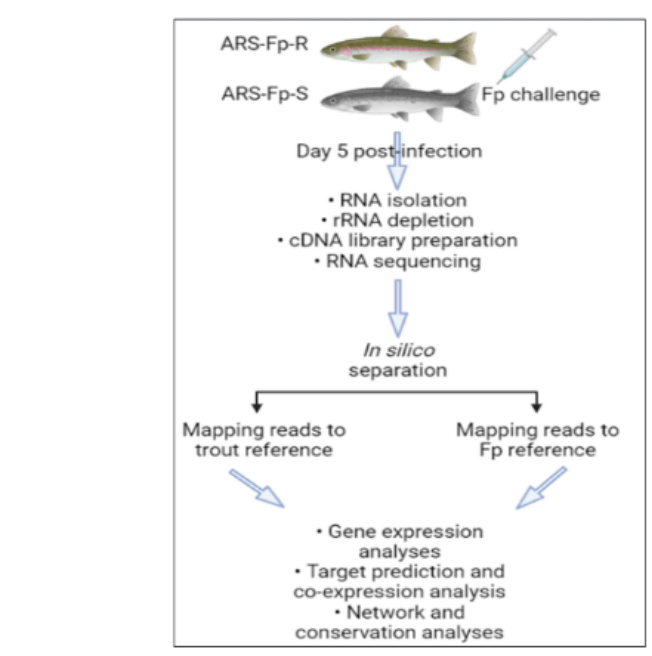

C

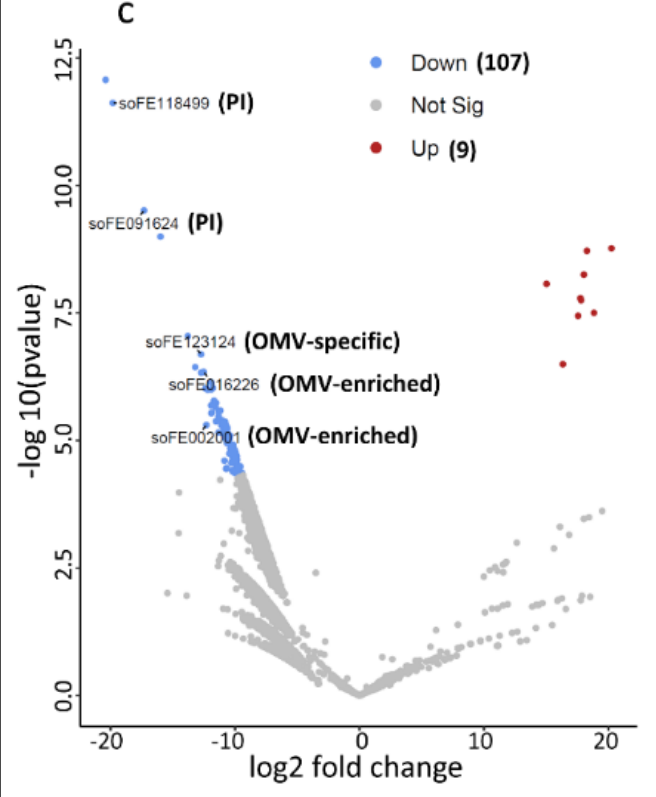

b
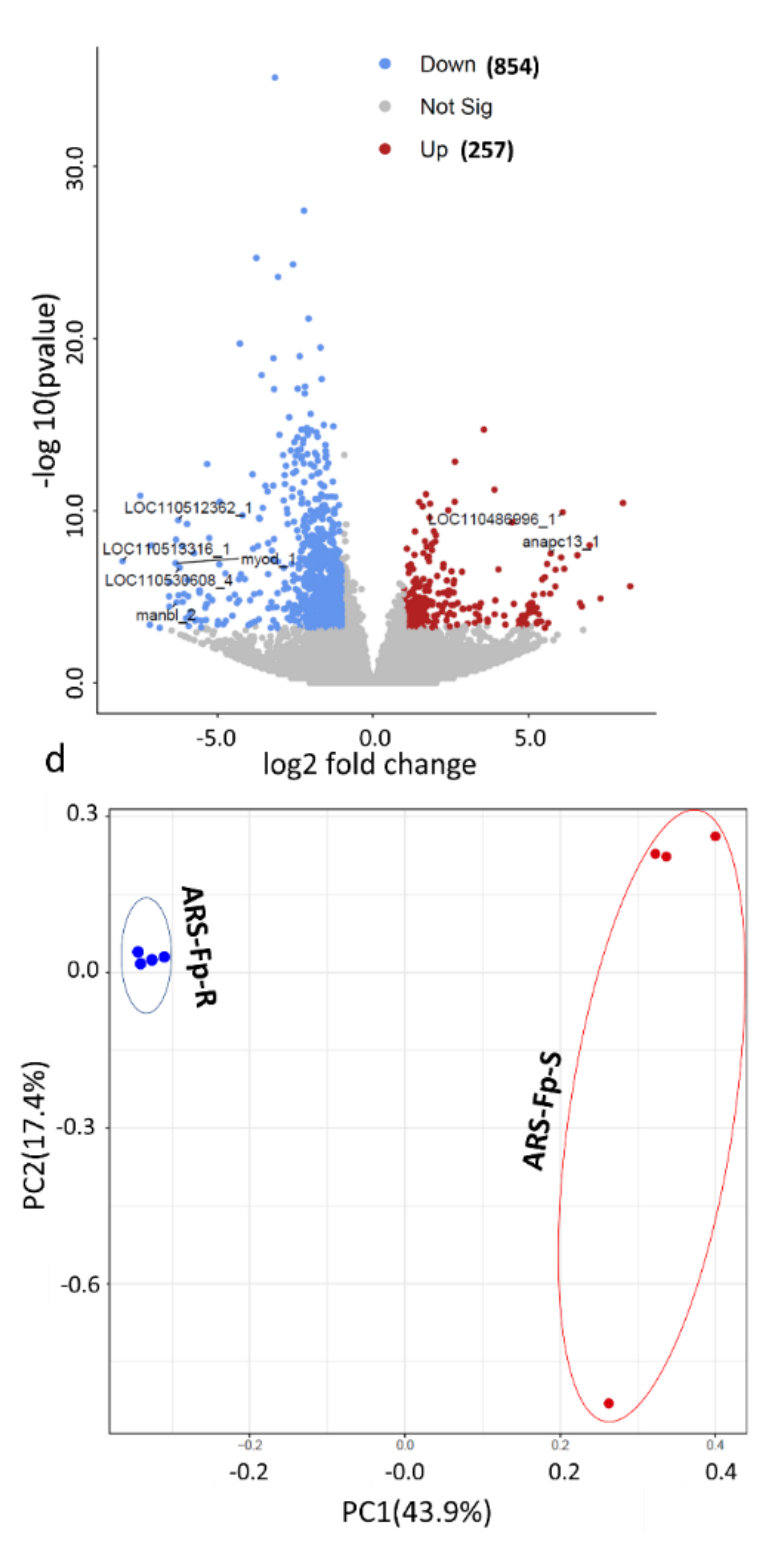

Fig. 5: a) Whole-body dual RNA-seq. Fish were intraperitoneally injected with $F$. psychrophilum as previously described by [6]. Total RNA was isolated from fish collected on day 5 post-infection and processed for sequencing. Sequence reads were separated, in silico, by mapping to the rainbow trout and $F$. psychrophilum genomes to identify DE transcripts and hub genes during host-pathogen interactions. b,c) Volcano plots showing the host transcripts (mRNAs $\&$ lncRNAs) and bacterial sRNAs, respectively, differentially expressed on day 5 following $F$. psychrophilum infection in selectively bred, resistant- versus susceptible-line rainbow trout. The red dots represent the upregulated transcripts in the resistant line, whereas the blue dots represent the downregulated transcripts at FDR $\leq 0.05$. Four sRNAs named on the figure, either located in the PAIs or enriched in OMVs, were among the most upregulated sRNAs in the susceptible genetic 
244 line. d) Principal component analysis of OMV-specific sRNAs obtained from 8 RNA-seq datasets 245 generated from selectively bred, resistant- and susceptible-line rainbow trout on day 5 post246 infection. Each round bullet represents a single RNA-seq dataset color-coded by a genetic line.

In order to gain insights into the implications and biological roles of bacterial sRNAs in fish

249 following infection, we profiled their expression in resistant and susceptible genetic lines (Fig. 5c)

250 and then investigated their expression correlation and interaction with the host genes (Fig. 6a and

251 Additional file 3; Tables S12 \& S13). Interestingly, a significant difference in the OMV-specific

252 sRNA expression was detected between the genetic lines on day 5 following infection (Fig. 5d).

253 A principal component analysis (Fig. 5d) showed that the primary axis accounts for $\sim 44 \%$ of the

254 variation. The pairwise comparison revealed 116 sRNAs with differential abundance between fish

255 from the two genetic lines following infection (Additional file 3; Table S11). Of them, 28 DE

256 sRNAs were OMV-specific. 107 sRNAs (92.2\%) were upregulated in susceptible fish. A total of

$25771 \mathrm{DE}$ sRNAs exhibited reciprocal expression correlation $(\mathrm{R}<-0.85)$ and interaction $(\mathrm{ndG} \leq-0.2)$

258 with 143 DE host protein-coding transcripts (Additional file 3; Table S12). For instance, the

259 OMV-specific sRNA soFE085997, followed by soFE129182 and soFE045225, exhibited the

260 highest potential of interaction with two isoforms encoding trichohyalin (Fig. 6b). The latter has

261 an essential role in the proliferation and anti-apoptosis of human keratinocytes [30], which

262 previously demonstrated a capacity to kill bacteria [31]. BCWD causes caudal lesions in rainbow

263 trout, and thus the upregulation of trichohyalin in resistant fish may represent a defense mechanism

264 against the pathogen. On the other hand, more DE bacterial sRNAs (98.3\%) were correlated with

265 DE host lncRNAs (70.6\%) in expression and were more likely to interact with each other (ndG $\leq$

266 -0.2) (Additional file 3; Table S13). Four of the hosts' lncRNAs showed expression correlation

267 and interaction with the host trichohyalin isoforms and bacterial sRNAs (Fig. 6b and Additional 268 file 3; Tables S13 \& S14). 

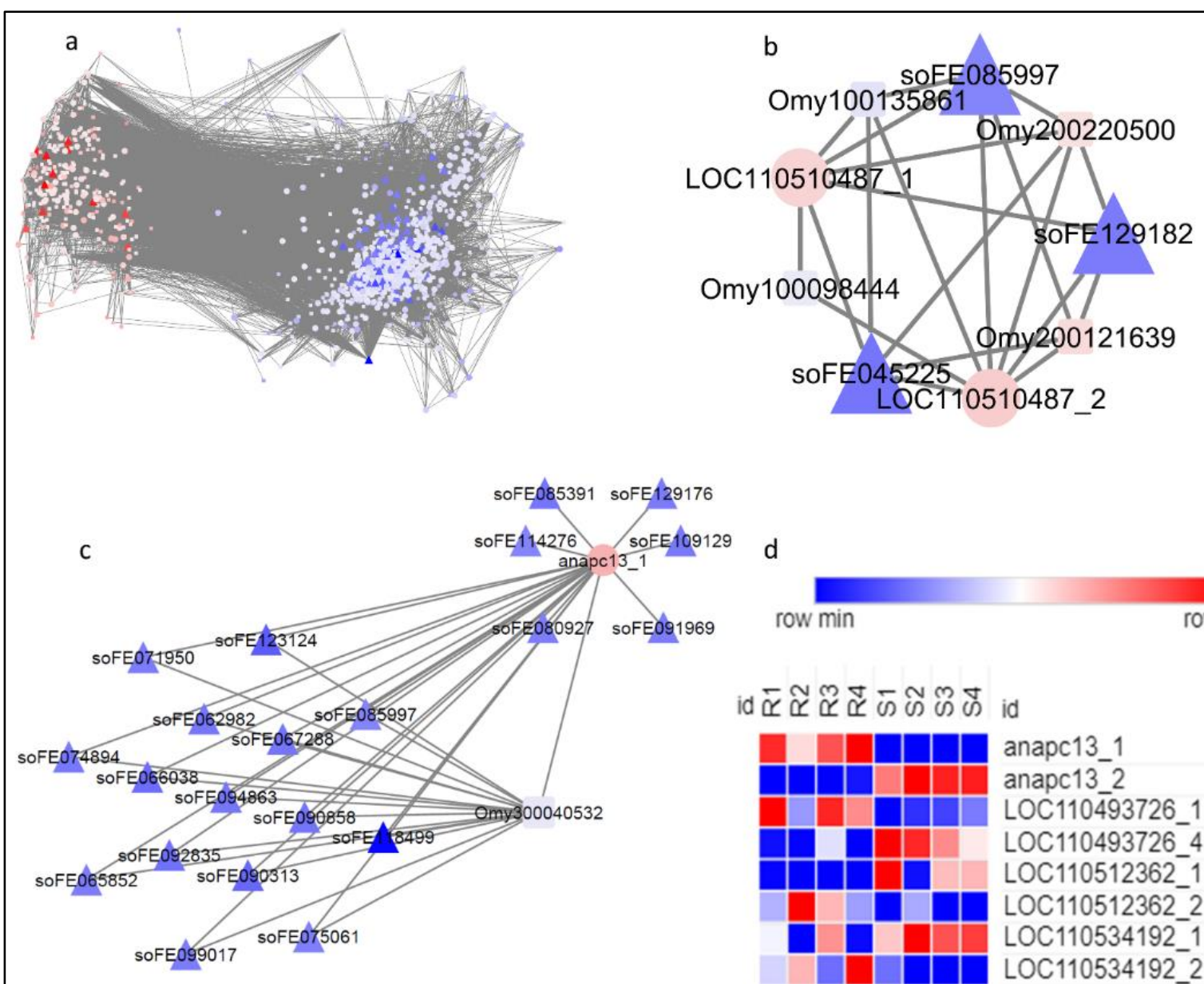

d

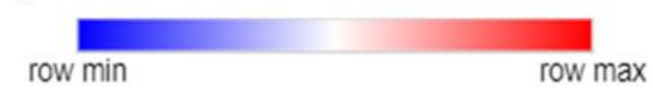

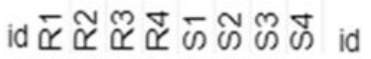

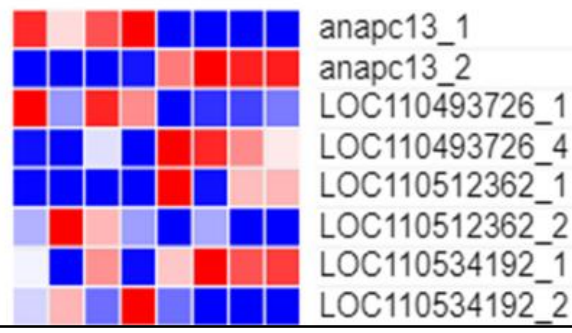

Fig. 6: a) Gene expression network of DE bacterial sRNAs (triangular nodes) and DE host transcripts (mRNAs "circular nodes" and lncRNAs "rectangular nodes") $(\mathrm{R}>0.85$ or $<-0.85)$. DE transcripts are clustered into two groups based on their fold change, with the upregulated transcripts in the BCWD-resistant genetic line represented in red and the downregulated transcripts represented in blue. b) Three bacterial sRNAs (blue), including the OMV-specific sRNA soFE085997, exhibited reciprocal expression with two isoforms encoding trichohyalin (LOC110510487_1 \& LOC110510487_2). c) ANAPC13_1 (red circle) was the top known downregulated transcript in susceptible fish on day 5 post-infection. ANAPC13_1 exhibited target interaction and negative correlation in expression with 21 sRNAs located in PAI or enriched in

280 OMVs. LncRNA Omy300040532 likely mediates the interaction between most of these sRNAs and ANAPC13_1. d) Four isoforms, including ANAPC13_1, exhibited reciprocal expression with 282 four other isoforms produced from the same genomic locus. "R1-4" refer to samples from the resistant genetic line, whereas "S1-4" refer to samples from the susceptible genetic line. 
Among the differentially regulated protein-coding transcripts, $445 \quad(86.2 \%)$ were

286 downregulated in the resistant line (Additional file 3; Table S9). The list included genes coding

287 for histone H3, MYOD protein, C-X-C motif chemokine 11, cathelicidin antimicrobial peptide,

288 and hepcidin. Histone $\mathrm{H} 3\left(\log _{2} \mathrm{FC}=-8.05\right)$ and MYOD $\left(\log _{2} \mathrm{FC}=-6.3\right)$ were at the top of the

289 downregulated genes in the resistant line. Pathogens, such as bacteria, reprogram the host cells

290 during infection through induction of histone $\mathrm{H} 3$ modifications, which modulate the host

291 transcription machinery [32]. Notably, nine sRNAs enriched in the parent bacterial cells exhibited

292 a strong reciprocal expression with histone H3. MYOD is a transcription factor involved in

293 activating genes coding for muscle-specific proteins [33, 34]. Inactivity or reduced swimming

294 activity has been recently reported to be associated with disease resistance in salmon [35]. In

295 Salmo salar, myosin was upregulated in susceptible fish in response to sea lice infection [35].

296 MYOD did not directly show correlation/interaction with the bacterial sRNAs. However, MYOD

297 showed a negative correlation $(\mathrm{R}=-0.88)$ with the lncRNA Omy200030291 (Additional file 3;

298 Table S14), which positively correlates and interacts with an OMV-specific sRNA soFE095462

299 located within the PAI (Additional file 3; Table S13).

$300 \quad$ In contrast, only 71 upregulated transcripts were identified in resistant fish (Additional file 3;

301 Table S9). Uncharacterized LOC110486996 and anaphase-promoting complex subunit 13

302 (ANAPC13_1) were the most highly upregulated transcripts. ANAPC13 is involved in cell cycle

303 progression and has been reported to have a potential role in class-I MHC-mediated antigen

304 presentation [36]. ANAPC13_1 exhibited interaction and reciprocal expression with 21 sRNAs

305 (Fig. 6c). Of them, the sRNA soFE1 18499 was located in PAI (Additional file 2; Table S6),

306 whereas 20 sRNAs were OMV-specific (Additional file 1; Table S3). Of note, most of the sRNAs

307 were more likely interacting with ANAPC13_1 through lncRNA Omy300040532, which 
308 negatively correlates in expression with ANAPC13_1 (Fig. 6c). Interestingly, eight coding

309 transcripts showed contrasting gene expressions (Fig. 6d). These transcripts encode ANAPC13,

310 UTP-glucose-1-phosphate uridylyltransferase, vicilin-like seed storage protein At2g18540, and

311 CCR4-NOT transcription complex subunit 6. Isoforms generated from the same gene locus have

312 previously been reported coding for antagonistic proteins. For instance, the fruit fly $B c l-x$ gene

313 generates two isoforms; one activates apoptosis while the other suppresses it [37].

314 Conservation of DE sRNAs in 65 strains with variable degree of virulence

315 We investigated the conservation of DE sRNAs in 65 strains with variable degrees of virulence.

316 Based on previous studies, 23 F. psychrophilum strains were categorized as high virulent strains,

317 and six strains were categorized as low virulent strains (see Methods section) [38-40]. Of 116 DE

318 sRNAs, seven DE sRNAs were conserved in all tested strains, whereas eight DE sRNAs were

319 present in the virulent strains. The list of sRNAs belonging to each group is included in Additional

320 file 3 (Table S15).

321 Among sRNAs present in virulent strains, a single sRNA (soFE015758) was located within a

322 PAI spanning the genomic region 583837-607648. Additionally, the OMV-specific sRNA

323 soFE030439 was conserved in virulent strains. Whereas sRNAs soFE031866, soFE036061, and

324 soFE026739 were enriched in OMVs compared to the whole-cell. sRNA soFE015758 targets

325 many immune genes, including dedicator of cytokinesis protein 2, leucine-rich repeats and

326 immunoglobulin-like domains protein 1, and tumor necrosis factor-alpha-induced protein 2.

327 Whereas sRNA soFE030439 strongly interacts with TNF superfamily member 5, myelin328 associated glycoprotein, and B-cell receptor CD22. 
In vitro treatment of RTgill-W1 cells with OMVs

330 A cell viability assay was performed to determine if OMVs cause lysis/death in RTgill-W1

331 cells after different time durations (Fig. 7a). A Presto Blue cell viability assay showed a significant

332 cell lysis/death after OMV exposure of 12 and 24 hours (Fig. 7b). The cytolytic effect of OMVs

333 might be mediated by the enzymes or toxins incorporated in OMVs.

334 To further understand the OMV-sRNA interaction with the host genes, we profiled

335 transcriptome expression of RTgill-W1 cells at 24h post-treatment with OMVs (Fig. 7c). RNA

336 sequencing yielded a total of 105,955,286 reads (Average 17,659,214 reads) from six cDNA

337 libraries prepared from control and treated cells (3 libraries/each). A total of 77,160,290 (72.82\%)

338 reads were mapped to the rainbow trout genome. 152 trout transcripts were DE in response to

339 OMV treatment (Fig. 7d and Additional file 3; Table S16). Most of them ( 66\%) were upregulated

340 in treated cells.

341 Surprisingly, our analysis of the RTgill-W1 cell response to OMV treatment after 24h,

342 considered an early response, did not reveal signs of downregulation of host genes with potential

343 roles in immunity. Only a single gene, complement factor $\mathrm{H}$ was downregulated in treated cells

344 (Additional file 3; Table S16). Pathogens exploit complement factor H to inhibit opsonization by

$345 \mathrm{C} 3$, and thus resist phagocytosis [41]. Conversely, we observed upregulated expression of several

346 immune-related genes such as complement protein component $\mathrm{C} 7-1$, chemokine $\mathrm{CXCF} 1 \mathrm{~b}$,

347 TNFAIP3-interacting protein 1, and transcription factor AP-1 (Additional file 3; Table S16). C7

348 molecule is a constituent of the membrane attack complex (MAC), which forms pores into

349 bacterial target membranes, leading to cell lysis and death [40].

350 The KEGG analysis provided a general overview of essential genes/pathways regulated

351 following OMV treatment. During the early response to OMVs, genes involved in chaperones and

352 folding catalysts, membrane trafficking and endocytic pathway, phagocytosis, and antigen 
353 processing and presentation mainly were upregulated (Fig. 7e-g and Additional file 3; Table S16).

354 Also, 12 transcripts encoding Hsp70, Hsp90, and cochaperones were upregulated in RTgill-

355 W1cells in response to OMV treatment (Fig. 7e).

356 In addition, some proteins involved in endosomal trafficking were upregulated, including Rab

357 and vacuolar protein sorting proteins. Contrariwise, we noticed downregulation of a few

358 endocytosis-related genes such as PH and SEC7 domain-containing protein 1, protein CLEC16A,

359 and SH3 domain-containing kinase binding protein 1 (sh3kbp1) (Fig. 7f). Fig. 7g shows seven

360 upregulated genes implicated in phagocytosis. The list includes genes essential for pathogen

361 recognition (CD209 molecule), actin remodeling (fibroblast growth factor 7 and transgelin-2), and

362 phagosome acidification (V-type proton ATPase catalytic subunit A; ATP6V1A).

363 Our results show early inhibition of the suppressor of cytokine signaling 1 at $24 \mathrm{~h}$ following

364 OMV treatment. Mycobacterium tuberculosis induced early expression of a member of the

365 suppressor of cytokine signaling (SOCS) family of proteins to control phagosomal acidification

366 by selectively targeting the ATP6V1A for degradation [42]. We also noticed downregulation of a

367 transcript encoding protection of telomeres 1 (POT1), which negatively regulates phagocytosis.

368 Additionally, ten upregulated genes involved in antigen processing and presentation were

369 identified. These genes encode beta-2-microglobulin (B2M) and nine heat shock proteins (Fig.

$3707 \mathrm{~g})$.

371 Furthermore, genes with anti-apoptotic roles, such as AP-1 proteins, DNA-damage-inducible

372 transcript 4 (DDIT4), heat shock proteins (Hsp70 and Hsp90), and cochaperones (DnaJ/Hsp40 and

373 tetratricopeptide repeat (TPR)), were upregulated (Additional file 3; Table S16). However, a few

374 genes whose products likely have apoptotic properties, such as MAP/microtubule affinity- 
regulating kinase 4 and Growth arrest and DNA-damage-inducible protein GADD45 beta,

376 exhibited contrasting expression (Additional file 3; Table S16).

377 Notably, 69 bacterial sRNAs showed significant expression (>100 reads) in the treated cells.

378 Of them, 13 sRNAs belong to the cell-enriched or OMV-enriched/-specific categories (Table 3).

379 The top four sRNAs targeted most of the downregulated host transcripts following OMV

380 treatment. For instance, the OMV-enriched sRNA soFE013584 exhibited a very high interaction

381 (ndG $=-0.54)$ with the transcript encoding POT1. Also, the OMV-enriched sRNA soFE002123

382 interacted with the POT1. POT1 protein is vital for the proper maintenance of genome integrity.

383 Disruption of POT1 function results in chromosome instability and loss of cellular viability [43].

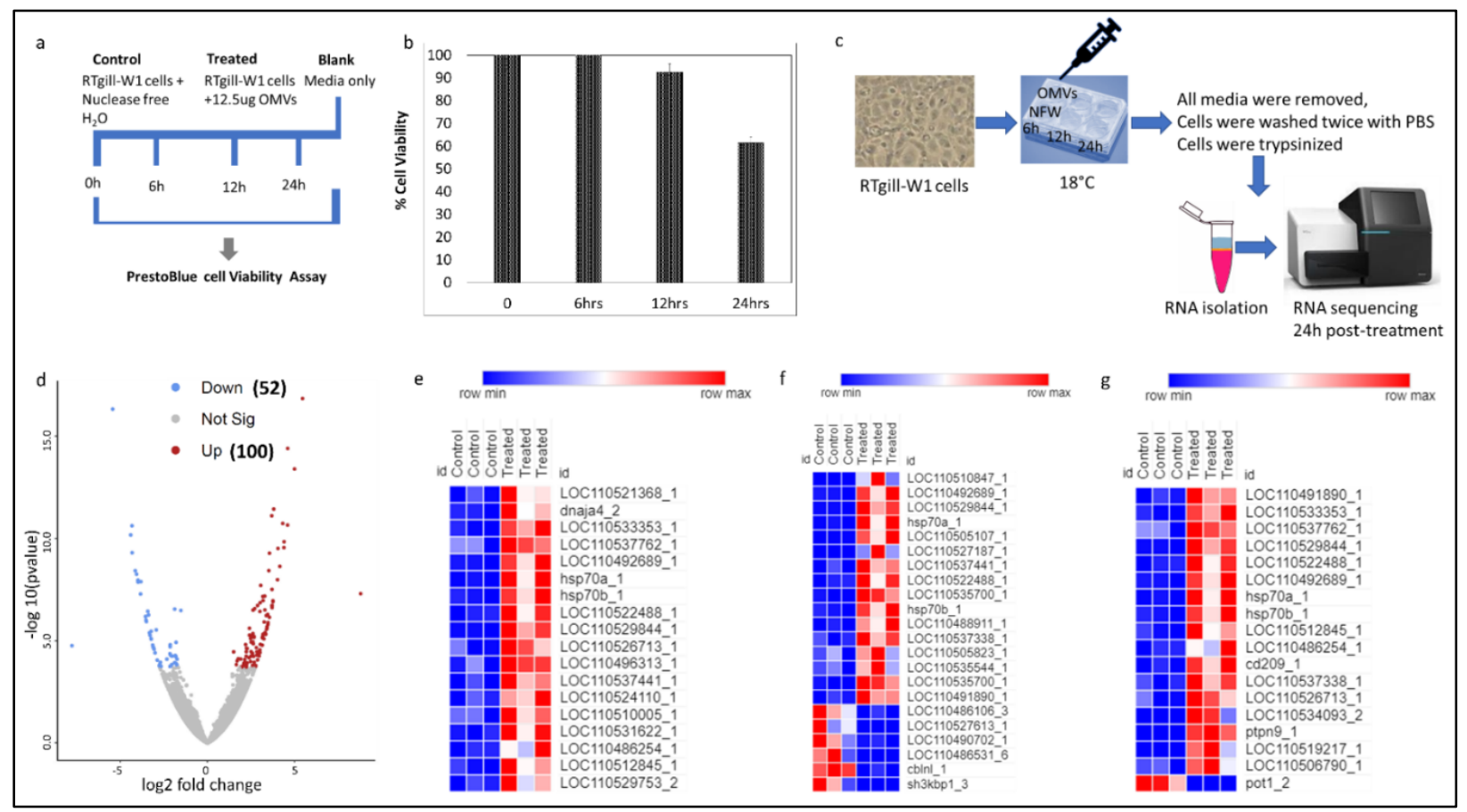

Fig. 7: a) Overview of PrestoBlue cell viability assay in RTgill-W1 cells after different time durations of OMVs treatment. Approximately 500,000 cells/well were seeded in 96-well plates for $24 \mathrm{~h}$. Cells were then treated with OMVs or Nuclease-free water (NFW; control group) for 0 min, $6 \mathrm{~h}, 12 \mathrm{~h}$, and $24 \mathrm{~h}$ at $18^{\circ} \mathrm{C}$. Cell culture medium was used as a blank for the PrestoBlue Assay. PrestoBlue reagent was added to wells, followed by incubation at appropriate temperatures and absorbance measurement at $570 \mathrm{~nm}$ to determine cell viability. b) PrestoBlue cell viability assay

390 in OMVs treated RTgill-W1 cells showed that the cell lysis/death increases with increased time 391 duration of OMVs exposure. Significant cell lysis was observed at 12 and 24h (p < 0.05). c) 
Approximately $1.2 \times 10^{6}$ RTgill-W1 cells/well were seeded in 6-well tissue culture plates and maintained overnight at $18^{\circ} \mathrm{C}$. Cells were exposed to either OMVs or NFW (control group) for $6 \mathrm{~h}, 12 \mathrm{~h}$, and $24 \mathrm{~h}$ at $18^{\circ} \mathrm{C}$. The media from the "control" and "treated" cell wells were removed, and cells were washed, trypsinized, and then lysed with Trizol reagent for RNA extraction and sequencing on day 1 post-treatment. d) A Volcano plot showing the host transcripts DE on day 1 following OMVs treatment in RTgill-W1 cells. The red dots represent the upregulated transcripts in the OMV-treated cells, whereas the blue dots represent the downregulated transcripts at FDR $\leq$ 0.05. Heat maps showing DE transcripts involved in chaperones and folding catalysts (e), membrane trafficking and endocytic pathway (f), and phagocytosis, and antigen processing and presentation (g).

Table 3: OMV-enriched/-specific versus whole cell-enriched bacterial sRNA detected 24h following treatment of the RTgill-W1 cells with OMVs. The negative log2 ratio indicates that sRNA is less abundant in OMV than the whole-cell.

\begin{tabular}{|c|c|c|c|c|c|}
\hline ID & Start & End & Read counts & Category (log2 ratio) & PAI \\
\hline soFE125269 & 1637553 & 1637893 & 3145 & cell-enriched (-1.72) & No \\
\hline soFE004028 & 2056951 & 2057269 & 2062 & OMV-specific & No \\
\hline SoFE013584 & 2196235 & 2196334 & 1912 & OMV-enriched (3.1) & No \\
\hline SoFE002123 & 2061751 & 2061850 & 1020 & OMV-enriched (4.23) & No \\
\hline soFE104747 & 1635059 & 1635287 & 918 & OMV-specific & No \\
\hline SoFE048173 & 2195010 & 2195109 & 657 & OMV-enriched (4.21) & No \\
\hline SoFE011466 & 597087 & 597258 & 272 & cell-enriched (-1.43) & Yes \\
\hline SoFE091624 & 595998 & 596220 & 264 & OMV-enriched (1.61) & Yes \\
\hline SoFE004713 & 1634302 & 1634689 & 231 & OMV-specific & No \\
\hline SoFE100359 & 593374 & 593473 & 216 & cell-enriched (-1.31) & Yes \\
\hline soFE073471 & 2540111 & 2540210 & 210 & OMV-enriched (1.63) & No \\
\hline soFE011096 & 2046508 & 2046607 & 181 & cell-enriched (-1.2) & No \\
\hline soFE100358 & 593129 & 593228 & 133 & OMV-specific & Yes \\
\hline
\end{tabular}

\section{Discussion}

409 Several studies have focused on the role of eukaryotic membrane vesicles carrying nucleic acid

410 content (DNA, RNA, miRNA) in disease states [44]. However, less is known about the role of

411 nucleic acids present within prokaryotic membrane vesicles in affecting the host during the host-

412 pathogen interactions. Previous studies have demonstrated the presence of DNA, peptidoglycan, 
413 and lipopolysaccharides (LPS) in OMVs purified from bacterial cultures [45, 46]. These cargos

414 can be delivered to host cells by binding OMVs to surface receptors on host cells or through

415 endocytosis $[47,48]$. Because OMVs provoke the transfer of various inner constituents from

416 bacteria to the host, it has driven our attention to further investigate the effect of OMVs on host-

417 pathogen interactions. Thus far, only a few studies [16, 49] have associated the presence of sRNAs

418 within bacterial OMVs with a role in host health. Dual RNA-seq identified sRNAs produced from

419 Salmonella enterica, such as PinT, demonstrated a role in regulating the expression of host genes

420 and mediating the activity of virulence genes necessary for intracellular survival of the pathogen

421 [50]. Koeppen et al. reported that P. aeruginosa sRNAs target kinases in the LPS-stimulated

422 MAPK signaling pathway [16]. sRNAs in the plant pathogen Xanthomonas compestris pv

423 vesicatoria were also involved in pathogenicity, and deletion of the sRNA led to a reduction in

424 virulence of bacteria [51].

425 In this study, RNA-Seq analysis revealed the presence of sRNAs in OMVs and found variation

426 in sRNA abundance between OMV and whole-cell transcriptome. Our results indicated that

427 OMV-specific/-enriched sRNAs target immune-relevant genes. These sRNAs included

428 soFE129980 (TPM 830), which targets the host NLR family CARD domain-containing protein 3;

429 soFE081095 which targets butyrophilin subfamily 1 member A1; and sRNA soFE023505, which

430 targets interferon-induced protein 44 and major histocompatibility complex class I-related gene

431 protein. Similar to our work, a recent study used RNA-Seq to characterize OMVs-packaged

432 sRNAs in $P$. aeruginosa and predicted their human immune transcript target. The study reported

433 a specific OMV-sRNA delivered to host cells, resulting in OMV-induced attenuation of IL-8

434 secretion and neutrophil infiltration in mouse lungs [16]. 
Although many attempts have been made to understand the mechanism of host-pathogen

436 interactions between rainbow trout and F. psychrophilum [6-8], very little is known regarding the

437 pathogenesis of this bacterium. Differential expression of trout genes between the ARS-Fp-R and

438 ARS-Fp-S genetic lines has been previously reported [6]. However, the potential influence of the

439 bacterial sRNAs in regulating the trout genes and their potential effect on the survivability of the

440 fish was not previously reported in rainbow trout. Our study showed that most DE sRNAs were

441 upregulated in fish susceptible to BCWD. OMV-enriched sRNAs exhibited strong interactions

442 and differential reciprocal expression with trout genes in BCWD-resistant and -susceptible genetic

443 lines. sRNAs interacting with the trout genes share complementary sequences, which ultimately

444 might cause post-transcriptional repression of the trout genes. sRNAs targeting immune genes

445 might help the bacteria to highjack the immune response and modulate the survival of bacteria in

446 the host [52]. The effect of sRNAs was tested in Staphylococcus aureus by silencing the sRNA,

447 which modulated the virulence and disease resistance of $S$. aureus [53]. Similarly, another study

448 in Staphylococcus reported the role of sRNA in bacterial virulence and regulating the expression

449 level of an immune evasion molecule [54]. Further investigation beyond the scope of this study is

450 required to determine whether deletion of specific bacterial sRNAs can affect the F. psychrophilum

451 invasion of the host. Identifying new microbial targets may facilitate controlling bacterial

452 infections and containing the BCWD.

453 The study revealed a significant cell lysis/death at 12 and 24 hours following in vitro exposure

454 of RTgill-W1 cells to $12.5 \mu \mathrm{g} / \mathrm{mL}$ OMVs. Consistently, Burkholderia cepacia OMVs induced

455 cytotoxicity in host cells treated with $\geq 10 \mu \mathrm{g} / \mathrm{mL}$ of OMVs for $24 \mathrm{~h}$ [55]. In addition, OMVs

456 induced cytotoxicity of the salmonid CHSE-214 cells [13]. Most of the DE transcripts involved

457 in cell death regulation are encoding products with anti-apoptotic roles, suggesting that cell death 
occurred by necrosis due to exposure to OMV toxins. One characteristic of the OMV treatment is

459 the upregulation of transcripts for HSP70, HSP90, and cochaperones. Cochaperones, such as

460 DnaJ/Hsp40 and tetratricopeptide repeat (TPR), determine the multifunctional properties of Hsp70

461 and Hsp90, including their anti-apoptotic functions [56]. The virulence factor Hsp60 was

462 identified in the proteome of OMVs of the fish pathogen Piscirickettsia salmonis[13]. In addition

463 to their role in regulating cell death/apoptosis, heat shock proteins stimulate elements of innate

464 immunity and traffic antigens into antigen-presenting cells, which facilitates induction of specific

465 acquired immune responses (reviewed in [57]).

466 Moreover, OMV treatment modulated host gene expression, favoring elements from the

467 phagocytic, endocytic, and antigen presentation pathways. Phagocytosis is a conserved defense

468 strategy in which host cells engulf and destroy self/nonself antigens. Although the epithelial

469 RTgill-W1 cells are not professional phagocytes, epithelial cells were previously reported to have

470 low phagocytic activity and a significant contribution to pathogen clearance [58]. Following OMV

471 treatment, RTgill-W1 cells upregulated the phagocytic receptor CD209 (DC-SIGN) responsible

472 for pathogen recognition [59]. Upregulation of genes involved in the regulation of actin

473 cytoskeleton, such as fibroblast growth factor and Transgelin-2, suggests that actin remodeling is

474 essential at the site of phagocytosis. It has been previously reported that Transgelin-2 [60] and

475 fibroblast growth factors [61, 62] enhance phagocytosis. Several other genes involved in

476 phagocytosis, including ATP6V1A, were also upregulated. V-ATPase molecules accumulate on

477 the phagosomal membrane to acidify the phagosome interior $[63,64]$. Some pathogenic bacteria

478 (M. tuberculosis) have developed a strategy to survive inside host phagocytes by facilitating

479 ATP6V1A degradation [42]. In addition to phagocytosis, genes implicated in the endocytic

480 pathway, such as early endosome antigen 1 (EAA1), vacuolar sorting proteins, and Rab proteins, 
481 were also regulated in response to OMV treatment. EAA1 is recruited to the phagosome,

482 promoting early endosomal fusion with the new phagosome [65].

483 Moreover, OMV-treated cells upregulated B2M, a scaffolding protein that retains the native

484 structure of MHC class I molecules on the surface of nucleated cells to present antigens to

485 cytotoxic $\mathrm{CD} 8+\mathrm{T}$ cells. In addition to the role of $\mathrm{B} 2 \mathrm{M}$ in adaptive immunity, it possesses

486 antimicrobial activities (i.e., innate response). B2M sheds sB2M-9 fragments, which function as

487 antibacterial chemokines, and perhaps as potential antimicrobial peptides (AMPs) following

488 modification by thioredoxin [66]. Upregulating the expression of phagocytosis-, endocytosis-, and

489 antigen presentation-related genes reflect host attempts to increase uptake of OMVs and present

490 processed antigens to stimulate the immune response.

491 OMVs possess some inherent characteristics that qualify them as vaccine candidates (reviewed

492 in [67]). For instance, OMVs are storage containers that can remain intact under different

493 treatments and temperatures. Also, OMVs have non-living activity, adjuvant effects, non-

494 replicative properties, and hold substantial immunogenic components belonging to the parent

495 bacteria, which may induce immune responses against bacterial infection. OMVs derived

496 from Salmonella Enteritidis [68] and Bordetella bronchiseptica [69] provided substantial

497 protection against the parent bacterial infection. However, further research is still needed to study

498 the role of F. psychrophilum-derived OMVs in boosting the host immune response, and thus their

499 potential application as a vaccine to contain/control the BCWD.

\section{Conclusions}

501 The current study provided the first characterization of $F$. psychrophilum sRNAs in OMVs and

502 demonstrated their potential effects on host gene expression in vivo and in vitro. One key aspect

503 of this study is the simultaneous capture of the RNA expression profile of the $F$. 
504 psychrophilum and its piscine host (rainbow trout) following infection in selectively bred resistant

505 and -susceptible genetic lines. The whole-body dual RNA-seq approach yielded a comprehensive

506 transcriptomic dataset that enabled us to identify microbial factors contributing to the disease

507 progression at a later stage of the infection. In particular, we identified bacterial sRNAs

508 packaged/enriched within OMVs and delivered to the host. These sRNAs revealed strong

509 interactions and reciprocal expression profiles with the host immune genes. Such a relationship

510 suggests a role for the OMV-enriched sRNAs in shaping the host-pathogen interactions and

511 assisting the bacteria to highjack the host immune response. Future studies considering the early

512 and late stages of infection will provide insights into the dynamics of gene expression changes

513 during infection.

514 Additionally, the study revealed significant cell lysis/death at 12 and 24 hours following in

515 vitro exposure of RTgill-W1 cells to OMVs. The OMV treatment also modulated host gene

516 expression, favoring elements from the phagocytic, endocytic, and antigen presentation pathways.

517 Further studies are needed to investigate whether OMVs can induce immune memory in rainbow

518 trout and thus assess their potential use as a vaccine.

519 Materials and Methods

520 Ethic Statement

521 Fish were maintained at the NCCCWA and animal procedures were performed under the

522 guidelines of NCCCWA Institutional Animal Care and Use Committee Protocols \#053 and \#076.

\section{Bacterial culture}

$524 \quad F$. psychrophilum (CSF 259-93) used in this study was obtained from USDA/NCCCWA (Dr.

525 Gregory Wiens). Frozen stock cultures of F. psychrophilum were cultured on Tryptone Yeast

526 Extracts (TYEs) agar, and the plate was incubated at $15^{\circ} \mathrm{C}$ for one week. F. psychrophilum

527 colonies isolated from the TYEs agar plate were transferred to TYEs broth, and absorbance 
528 (525nm) was measured after $24 \mathrm{~h}$ of incubation. Absorbance was measured every day for 2 weeks

529 to determine the log phase of the cultures. TYEs broth culture without $F$. psychrophilum was used

530 as a negative control.

531 Isolation of OMVs

532 OMVs were isolated from F. psychrophilum broth culture on day 8 of bacterial growth. A

533 loopful of culture was sub-cultured onto a plate on day 7 of bacterial growth to ensure the broth

534 was free of contamination. For OMV isolation, broth culture from a flask was distributed into

535 several $50 \mathrm{ml}$ tubes. Each tube was centrifuged at $2800 \mathrm{X} \mathrm{g}$ for $1 \mathrm{~h}$ at $4^{\circ} \mathrm{C}$ to pellet the bacterial

536 cells. The supernatant was collected and filtered through a $250 \mathrm{ml}$ sterile $0.22 \mu \mathrm{m}$ PES membrane

537 filter (EMD Millipore Corporation, Billerica, MA, USA) to remove any remaining bacterial cells.

538 The filtrate was then subjected to ultracentrifugation for $3 \mathrm{~h}$ at $40,000 \mathrm{rpm}$ and $4^{\circ} \mathrm{C}$ to pellet the

539 OMVs. The OMV pellet was washed with phosphate-buffered saline (PBS) and again subjected

540 to ultracentrifugation for $2 \mathrm{~h}$ at $40,000 \mathrm{rpm}$ and $4^{\circ} \mathrm{C}$ to re-pellet the OMVs. The OMV pellet was

541 resuspended in nuclease-free water and stored at $-20^{\circ} \mathrm{C}$. The protein concentration of the OMVs

542 was quantified using a BCA Protein assay kit (Thermo Fisher Scientific). To ensure that the

543 suspension containing OMVs was free of bacteria, $30 \mu 1$ of the suspension was cultured on a TYEs

544 agar plate and incubated for 10 days.

545 Transmission Electron Microscopy (TEM)

546 Transmission electron microscopy was performed on F. psychrophilum cell and OMV

547 samples. For F. psychrophilum cells, a single colony from a TYEs-agar plate was suspended in

548 nuclease-free water. Water and TYEs broth were used as negative controls. All samples were

549 subjected to negative staining using uranyl acetate, and samples were deposited on a TEM Carbon

550 coated grids (80 mesh square grid, EMS; Ted Pella, Inc., Redding, CA, USA) and incubated for 2 
551 min. Samples were blotted dry, and grids were washed with sterile deionized water $\left(\mathrm{diH}_{2} \mathrm{O}\right)$ three

552 times (30 seconds each) to remove the salt buffer. Excess water from grids was removed with

553 blotting paper before the samples were stained. Samples were stained with $5 \mu 1$ of $1 \%$ uranyl

554 acetate added onto the grid and incubated for about 1 min. The stain was then washed with sterile

$555 \mathrm{diH}_{2} \mathrm{O}$, dried, and the grids were observed under a Hitachi H-7650-II (Schaumburg, IL, USA)

556 transmission electron microscope.

Bacterial RNA extraction, cDNA Library Preparation, and Sequencing

$558 \quad$ RNA was extracted from F. psychrophilum colonies isolated from a TYEs agar plate and from

559 OMVs isolated from F. psychrophilum broth culture using TriZol reagent (Invitrogen, Carslsbad,

560 CA, USA). The RNA concentration was measured using a Nanodrop ${ }^{\mathrm{TM}}$ ND-1000

561 spectrophotometer (Thermo Fisher Scientific, Waltham, MA, USA). To confirm the existence of

562 RNA in OMVs, $4 \mu \mathrm{l}$ of the RNA samples were treated with $2 \mu \mathrm{g} / \mathrm{ul}$ of RNase Cocktail Enzyme

563 mix (Thermo Fisher Scientific Baltics, VA) and incubated in a water bath at $37^{\circ} \mathrm{C}$ for $30 \mathrm{~min}$.

564 RNase-treated and -untreated OMV RNA samples were then run on an agarose gel. RNA samples

565 isolated from $F$. psychrophilum and OMVs were stored at $-80^{\circ} \mathrm{C}$ until further processing.

566 For library preparation and RNA sequencing, RNA samples isolated from $F$. psychrophilum

567 cells and OMVs were sent to BGI Genomics (Cambridge, MA, USA). Library preparation was

568 performed using a Trio RNA-Seq kit (NuGEN, San Carlos, CA, USA) according to manufacturer

569 recommendations. Briefly, rRNA was depleted and RNAs were fragmented using a fragmentation

570 buffer. Fragmented pieces were then purified using QiaQuick PCR extraction kit (QIAGEN,

571 Germantown, MD, USA), and the solution was resuspended in EB buffer, and cDNA was

572 subjected to end repair and poly (A) tail addition. The fragments were then connected with the

573 adaptors. The library was then subjected to purification using a MiniElute PCR Purification kit 
574 before PCR amplification. PCR was used to amplify the libraries, and then the yield was

575 quantified. Sequencing was performed on an Illumina MiSeq platform (Illumina, Inc., San Diego,

576 CA, USA). Raw RNA-Req reads were submitted to the NCBI Short Read Archive under accession

577 number BioProject ID PRJNA259860 (accession number SUB10837562).

578 Prediction of sRNAs and their target genes

579 Raw sequence reads were trimmed by removing adaptor sequences and 5 bp from each end,

580 followed by filtering out low-quality reads. High-quality reads from F. psychrophilum

$581(60,352,578$ reads $)$ and OMVs (55,722,742 reads) were used for downstream analyses. Reads

582 from the $F$. psychrophilum transcriptome and OMVs were mapped to the $F$. psychrophilum (CSF-

583 259-93) reference genome using TopHat2 [70]. The FlaiMapper tool [28] was used to identify

584 potential sRNAs. sRNAs were predicted and filtered according to their length. sRNAs greater

585 than 500nt long were filtered out. DE transcripts and trout immune-related genes targeted by the

586 DE sRNAs were predicted using a locally installed LncTar software [71]. A normalized deltaG

587 (ndG) of -0.10 was used as a cutoff value to determine the potential interaction among paired

588 RNAs.

$590 \quad$ FISH staining was used to confirm the presence of sRNAs within F. psychrophilum. Briefly,

591 F. psychrophilum cultures from the log phase were harvested and centrifuged at $2800 \mathrm{rpm}$ for 5

$592 \min$ at $4^{\circ} \mathrm{C}$. The supernatant was discarded and the pellet containing the cells was washed with

593 PBS and then fixed with $4 \%$ paraformaldehyde in PBS for $4 \mathrm{~h}$ at $4^{\circ} \mathrm{C}$. After fixation, $10 \mu \mathrm{l}$ of the

594 cells were smeared onto a microscopic glass slide, and the smear was air-dried. Cells were then

595 dehydrated in an alcohol series (50, 80, and 100\% ethanol) for $3 \mathrm{~min}$ in each concentration at room

596 temperature. Cells were then hybridized with a Cy3 fluorophore-conjugated sRNA probe 
597 (soFE128978) for $2 \mathrm{~h}$ at $46^{\circ} \mathrm{C}$ in the dark. A non-sense eubacterial probe and a sense eubacterial

598 probe with FAM fluorophore were used as negative and positive controls. To prevent drying,

599 slides were incubated in a hybridization chamber. After hybridization, cells were washed and then

600 incubated with washing buffer $(5 \mathrm{M} \mathrm{NaCl}, 1 \mathrm{M}$ Tris/HCl, $0.5 \mathrm{M}$ EDTA, $10 \%$ SDS and distilled

$\left.601 \mathrm{H}_{2} \mathrm{O}\right)$ at $48^{\circ} \mathrm{C}$ for $25 \mathrm{~min}$. Then cells were incubated for $3 \mathrm{~min}$ in PBS containing DAPI (300 nM).

602 DAPI-stained slides were then washed with water and air-dried. Glass coverslips were mounted

603 to each slide after adding a drop of VectaShield (Vector Laboratories, Burlingame, CA, USA); the

604 edges of the coverslip were sealed with clear nail polish. Once dried, the slides were observed

605 using a Zeiss Axio Observer microscope with LSM700 confocal module. Imaging was performed

606 with ZEN 2012 software (Black Edition, Carl Zeiss Microscopy, Thornwood, NY, USA). Smart

607 Setup function of the ZEN 2012 imaging software was used to assign the optimal filter and beam

608 splitter settings for each laser while laser power and gain were set manually.

609 BCWD-resistant and -susceptible fish population, and bacterial challenge

610 The current study used samples from two rainbow trout genetic lines of divergent resistance to

611 BCWD. USDA-NCCCWA produced these genetic lines via a family-based selection method as

612 previously described [72]. Briefly, single-sire $\times$ single-dam matings were made between 3-year-

613 old dams and 1-year old sires (neo-males) within the genetic lines. The resistant line eggs were

614 produced from dams that had undergone three generations of BCWD selection, whereas the sires

615 had undergone four generations of selection to increase the disease resistance phenotype. On the

616 other hand, the susceptible line eggs were produced from parents that had undergone one

617 generation of selection to increase susceptibility to infection. The resistant and susceptible genetic

618 lines had significant differences in susceptibility to F. psychrophilum [6, 7]. 
For this study, fish from resistant and susceptible genetic lines (49 days post-hatch) were

620 challenged with F. psychrophilum as we previously described [6]. Briefly, fifty fish were

621 randomly assigned to each tank with $2.4 \mathrm{~L}$ min-1 of $12.5 \pm 0.1{ }^{\circ} \mathrm{C}$ flow-through spring water

622 supply. For each genetic line, two fish tanks were intraperitoneally injected with $4.2 \times 106$ CFU

623 fish-1 F. psychrophilum suspended in $10 \mu \mathrm{PBS}$, and survival was monitored daily for 21 days.

624 Five individuals were sampled from each tank on day 5 post-injection. All fish were certified to

625 be infection-free before being injected with F. psychrophilum.

626 RNA sequencing and gene expression analysis of BCWD-resistant and -susceptible fish

627 As explained above, tissue samples used in this study were obtained from USDA/NCCCWA

628 (Dr. Gregory D Wiens). RNA was extracted from the whole fish using TriZol, followed by quantity

629 and quality assessments as described above. RNA samples were then treated with DNAase I

630 (Fisher BioReagents, Hudson, NH, USA) to remove DNA from samples. For sequencing, equal

631 amounts of RNA samples were pooled from 2 fish, and 4 pooled samples were sequenced from

632 each of the resistant and susceptible genetic lines (i.e., a total of 8 libraries). Sequencing was done

633 at RealSeq Biosciences, Inc. (Santa Cruz, CA, USA). The Zymo Ribofree library prep kit targeted

634 all RNAs during the library preparation, including trout and bacterial RNAs.

635 Raw sequence reads from each genetic line were trimmed to obtain high-quality reads. Read

636 mapping and gene expression analyses were performed using a CLC genomics workbench. Reads

637 were separately mapped to the rainbow trout genome [USDA OmykA_1.1 assembly

638 (GCF_013265735.2)] and F. psychrophilum reference genomes to identify DE transcripts.

639 Mapping criteria were as follows: mismatch $\operatorname{cost}=2$, insertion/deletion $\operatorname{cost}=3$, minimum length

640 fraction $=0.9$, and similarity fraction $=0.9$. The expression value of each transcript was calculated

641 in terms of TPM and DE transcripts between resistant and susceptible genetic lines were identified 
642 using EDGE test (FDR value $<0.05, \log 2$ fold change cutoff \pm 1 ). Raw RNA-Req reads were

643 submitted to the NCBI Short Read Archive under accession number BioProject ID PRJNA259860

644 (accession number SUB10837562).

645

646

647 Collection, Manassas, VA, USA). Cells were handled according to instructions provided by

648 ATCC. Briefly, frozen cells were thawed at $20^{\circ} \mathrm{C}$ in a water bath, and the full content from the

649 vial was transferred to L-15 media supplemented with 10\% complement inactivated fetal bovine

650 serum (FBS) and 100 units/ml of penicillin and $100 \mu \mathrm{g} / \mathrm{ml}$ of streptomycin (complete medium).

651 Cells were centrifuged at $125 \mathrm{X}$ g for $8 \mathrm{~min}$, the supernatant was removed, and the cells were

652 resuspended in a complete medium. The cells were maintained in $25 \mathrm{~cm} 2$ flasks at $18^{\circ} \mathrm{C}$. Media

653 were renewed every 4 days, and cells were passaged every 8 days. Since cells are adherent, cells

654 were dislodged by trypsinization while passaging.

\section{Exposure of RTgill-W1 cells to OMVs}

Approximately $1.2 \times 10^{6}$ RTgill-W1 cells/well in L-15 media supplemented with $2 \%$ FBS [73]

were seeded in 6-well tissue culture plates. Before treatment, cells were maintained overnight at $18^{\circ} \mathrm{C}$. A $100 \mu \mathrm{l}$ volume of OMVs (total protein concentration $=0.8 \mathrm{mg}$ ) was added to cells for $6 \mathrm{~h}$,

660 triplicate. Plates were incubated at $18^{\circ} \mathrm{C}$, and sampling was done after each incubation period. All

661 the media from the "control" and "treated cells" were removed, and cells were then washed twice

662 with PBS. The cells were trypsinized and then lysed with Trizol reagent (Invitrogen, Carslsbad,

663 CA, USA) for RNA extraction, followed by sequencing and gene expression analyses. To 664 determine the cell viability, we performed a PrestoBlue Assay in which $~ 500,000$ cells/well were 
665 seeded in a 96 well plate for $24 \mathrm{~h}$. Cells were then treated with OMVs for different time intervals

666 of $0 \mathrm{~min}, 6 \mathrm{~h}, 12 \mathrm{~h}$, and $24 \mathrm{~h}$. Each condition was run in triplicate. Nuclease-free water was added

667 to cells as a control, and only cell culture medium was used as a blank for the PrestoBlue Assay.

668 After treatment, PrestoBlue reagent was added to wells, and then the plate was incubated at $37^{\circ} \mathrm{C}$

669 for $10 \mathrm{~min}$ followed by incubation at $18^{\circ} \mathrm{C}$ for $1 \mathrm{~h}$. The absorbance of treated cells and controls

670 was then measured at $570 \mathrm{~nm}$ to determine cell viability.

671 sRNAs conservation and identification of sRNAs within pathogenicity Islands

672 To determine the conservation of sRNAs, DE sRNAs reported in this study were blasted

673 against the genome sequences of 65 different $F$. psychrophilum strains downloaded from the

674 National Center for Biotechnology Information (NCBI) GenBank database

675 (https://www.ncbi.nlm.nih.gov/genome/browse/\#!/prokaryotes/1589/). F. psychrophilum strains

676 used in this study were 950106-1/1, DSM 3660, 010418-2/1, 4, 10, 11754, 17830, 160401-1/5,

677 990512-1/2A, CH8, CH1895, CHN6, CN, CR, F164, FI055, FI070, FPG3, FPG48, FPRT1, FPG1,

678 FPS-F15, 160401-1/5N, P30-2B/09, F164, FPS-F16, FPS-S11A, 990512-1/2A, P15-8B/11,

679 160401-1/5M, FPS-F27, FPS-R7, FPS-S9, JIP02-86, FPS-S6, OSU THCO2-90, FPS-P3, FPS-P1,

680 V46, JIP 08/99, JIP 16/00, FPS-S10, 010418-2/1, K9/00, 950106-1/1 , FPG3, FPGIW08, IT02,

681 IT09, IWL08, K9/00, KKOK-1706, KU060626-59, LM01-Fp, MH1, NNS1-1804, NNS4-1804,

682 NO014, OSU-THCO2-90, P7-7B/10, P158B-11, P30-2B, TN, TR). The high virulent Fp strains

683 included FPG1, FPS-F15, 160401-1/5N, P30-2B/09, F164, FPS-F16, FPS-S11A, 990512-1/2A,

684 P15-8B/11, 160401-1/5M, FPS-F27, FPS-R7, FPS-S9, JIP02-86, FPS-S6, OSU THCO2-90, FPS-

685 P3, FPS-R9, FPS-P1, V46, JIP 08/99, JIP 16/00) [40], whereas less virulent Fp strains included

686 FPS-S10, 010418-2/1, K9/00, 950106-1/1, FPG3, and FPGIW08 [40, 74]. A threshold of >95\%

687 identity and $100 \%$ query coverage were used as the cutoff values for homology. 
to identify and visualize pathogenicity islands present within the F. psychrophilum genome

sequence. Bedtools [76] was used to determine the location of sRNAs within the islands.

\section{References}

694 1. Nematollahi A, Decostere A, Pasmans F, Haesebrouck F. Flavobacterium psychrophilum infections

695 in salmonid fish. J Fish Dis. 2003;26(10):563-74. Epub 2003/12/05. doi: 10.1046/j.1365696 2761.2003.00488.x. PubMed PMID: 14653314.

$6972 . \quad$ Starliper CE. Bacterial coldwater disease of fishes caused by Flavobacterium psychrophilum. 698 Journal of Advanced Research. 2011;2: 97-108.

699 3. Barnes ME, Brown ML, . . A review of Flavobacterium psychrophilum biology, clinical signs, and 700 bacterial cold water disease prevention and treatment. The Open Fish Science Journal 2011;4:1-9.

701 4. Madetoja. J, Hänninen. M-L, Dalsgaard. VH-KI, Wiklund. T. Phenotypic and genotypic 702 characterization of Flavobacterium psychrophilum from Finnish fish farms.: Journal of Fish Diseases; 2001. 703 p. 469-79.

704 5. Gomez E, Mendez J, Cascales D, Guijarro JA. Flavobacterium psychrophilum vaccine development: 705 a difficult task. Microb Biotechnol. 2014;7(5):414-23. Epub 20140723. doi: 10.1111/1751-7915.12099. PubMed PMID: 25056179; PubMed Central PMCID: PMCPMC4229322.

6. Marancik D, Gao G, Paneru B, Ma H, Hernandez AG, Salem M, et al. Whole-body transcriptome of selectively bred, resistant-, control-, and susceptible-line rainbow trout following experimental challenge with Flavobacterium psychrophilum. Front Genet. 2014;5:453. Epub 20150108. doi: 10.3389/fgene.2014.00453. PubMed PMID: 25620978; PubMed Central PMCID: PMCPMC4288049.

7. Paneru B, Al-Tobasei R, Palti Y, Wiens GD, Salem M. Differential expression of long non-coding RNAs in three genetic lines of rainbow trout in response to infection with Flavobacterium psychrophilum. Sci Rep. 2016;6:36032. Epub 20161027. doi: 10.1038/srep36032. PubMed PMID: 27786264; PubMed Central PMCID: PMCPMC5081542.

8. Ali A, Thorgaard GH, Salem M. PacBio Iso-Seq Improves the Rainbow Trout Genome Annotation and Identifies Alternative Splicing Associated With Economically Important Phenotypes. Front Genet. 2021;12(1194):683408. Epub 20210715. doi: 10.3389/fgene.2021.683408. PubMed PMID: 34335690; PubMed Central PMCID: PMCPMC8321248.

9. Wiens GD, LaPatra, S. E., Welch, T. J., Evenhuis, J. P., Rexroad, C. E. III., and Leeds, T. D. On-farm performance of rainbow trout (Oncorhynchus mykiss) selectively bred for resistance to bacterial cold water disease: effect of rearing environment on survival phenotype. j.aquaculture; 2013. p. 388-91, 12836.

723 10. Barbier P, Rochat T, Mohammed HH, Wiens GD, Bernardet JF, Halpern D, et al. The Type IX Environ Microbiol. 2020;86(16). Epub 20200803. doi: 10.1128/AEM.00799-20. PubMed PMID: 32532872; PubMed Central PMCID: PMCPMC7414955. 
11. Moller JD, Barnes AC, Dalsgaard I, Ellis AE. Characterisation of surface blebbing and membrane vesicles produced by Flavobacterium psychrophilum. Dis Aquat Organ. 2005;64(3):201-9. Epub 2005/07/07. doi: 10.3354/dao064201. PubMed PMID: 15997818. 12. Ellis TN, Kuehn MJ. Virulence and immunomodulatory roles of bacterial outer membrane vesicles. Microbiol Mol Biol Rev. 2010;74(1):81-94. Epub 2010/03/04. doi: 10.1128/MMBR.00031-09. PubMed PMID: 20197500; PubMed Central PMCID: PMCPMC2832350.

13. Oliver C, Valenzuela K, Hernandez M, Sandoval R, Haro RE, Avendano-Herrera R, et al. Characterization and pathogenic role of outer membrane vesicles produced by the fish pathogen Piscirickettsia salmonis under in vitro conditions. Vet Microbiol. 2016;184:94-101. Epub 20151107. doi: 10.1016/j.vetmic.2015.09.012. PubMed PMID: 26854350.

14. Haurat MF, Aduse-Opoku J, Rangarajan M, Dorobantu L, Gray MR, Curtis MA, et al. Selective sorting of cargo proteins into bacterial membrane vesicles. J Biol Chem. 2011;286(2):1269-76. Epub 20101105. doi: 10.1074/jbc.M110.185744. PubMed PMID: 21056982; PubMed Central PMCID: PMCPMC3020734.

15. Jan AT. Outer Membrane Vesicles (OMVs) of Gram-negative Bacteria: A Perspective Update. Front Microbiol. 2017;8:1053. Epub 20170609. doi: 10.3389/fmicb.2017.01053. PubMed PMID: 28649237; PubMed Central PMCID: PMCPMC5465292. 16. Koeppen K, Hampton TH, Jarek M, Scharfe M, Gerber SA, Mielcarz DW, et al. A Novel Mechanism of Host-Pathogen Interaction through SRNA in Bacterial Outer Membrane Vesicles. PLoS Pathog. 2016;12(6):e1005672. Epub 20160613. doi: 10.1371/journal.ppat.1005672. PubMed PMID: 27295279; PubMed Central PMCID: PMCPMC4905634.

17. van der Pol L, Stork M, van der Ley P. Outer membrane vesicles as platform vaccine technology. Biotechnol J. 2015;10(11):1689-706. Epub 2016/02/26. doi: 10.1002/biot.201400395. PubMed PMID: 26912077; PubMed Central PMCID: PMCPMC4768646. 2018:6(2). Epub 2018/04/07. doi: 101128/microbiolspec.RWR-0004-2017. PubMed PMID. 29623872. PubMed Central PMCID: PMCPMC5890949.

19. Chao Y, Papenfort K, Reinhardt R, Sharma CM, Vogel J. An atlas of Hfq-bound transcripts reveals 3' UTRs as a genomic reservoir of regulatory small RNAs. EMBO J. 2012;31(20):4005-19. Epub 20120824. doi: 10.1038/emboj.2012.229. PubMed PMID: 22922465; PubMed Central PMCID: PMCPMC3474919.

20. Padalon-Brauch G, Hershberg R, Elgrably-Weiss M, Baruch K, Rosenshine I, Margalit H, et al. Small RNAs encoded within genetic islands of Salmonella typhimurium show host-induced expression and role in virulence. Nucleic Acids Res. 2008;36(6):1913-27. Epub 20080211. doi: 10.1093/nar/gkn050. PubMed PMID: 18267966; PubMed Central PMCID: PMCPMC2330248.

21. Gottesman S, Storz G. Bacterial small RNA regulators: versatile roles and rapidly evolving variations. Cold Spring Harb Perspect Biol. 2011;3(12). Epub 20111201.2 doi: 10.1101/cshperspect.a003798. PubMed PMID: 20980440; PubMed Central PMCID: PMCPMC3225950.

22. Wang J, Rennie W, Liu C, Carmack CS, Prevost K, Caron MP, et al. Identification of bacterial sRNA regulatory targets using ribosome profiling. Nucleic Acids Res. 2015;43(21):10308-20. Epub 20151105. doi: 10.1093/nar/gkv1158. PubMed PMID: 26546513; PubMed Central PMCID: PMCPMC4666370.

23. Kang XM, Wang FF, Zhang H, Zhang Q, Qiana W. Genome-wide identification of genes necessary for biofilm formation by nosocomial pathogen Stenotrophomonas maltophilia reveals that orphan response regulator FsnR is a critical modulator. Appl Environ Microbiol. 2015;81(4):1200-9. Epub 2014/12/07. doi: 10.1128/AEM.03408-14. PubMed PMID: 25480754; PubMed Central PMCID: PMCPMC4309692.

24. Chen XP, Ali L, Wu LY, Liu C, Gang CX, Huang QF, et al. Biofilm Formation Plays a Role in the Formation of Multidrug-Resistant Escherichia coli Toward Nutrients in Microcosm Experiments. Front 
Microbiol. 2018;9:367. Epub 20180302. doi: 10.3389/fmicb.2018.00367. PubMed PMID: 29552003; PubMed Central PMCID: PMCPMC5840168.

25. Chen R, Wei X, Li Z, Weng Y, Xia Y, Ren W, et al. Identification of a small RNA that directly controls the translation of the quorum sensing signal synthase gene rhll in Pseudomonas aeruginosa. Environ Microbiol. 2019;21(8):2933-47. Epub 20190610. doi: 10.1111/1462-2920.14686. PubMed PMID: 31106966.

26. Mader U, Nicolas $P$, Depke $M$, Pane-Farre J, Debarbouille $M$, van der Kooi-Pol MM, et al. Staphylococcus aureus Transcriptome Architecture: From Laboratory to Infection-Mimicking Conditions. PLoS Genet. 2016;12(4):e1005962. Epub 20160401. doi: 10.1371/journal.pgen.1005962. PubMed PMID: 27035918; PubMed Central PMCID: PMCPMC4818034.

27. Papenfort K, Bassler BL. Quorum sensing signal-response systems in Gram-negative bacteria. Nat Rev Microbiol. 2016;14(9):576-88. Epub 2016/08/12. doi: 10.1038/nrmicro.2016.89. PubMed PMID: 27510864 ; PubMed Central PMCID: PMCPMC5056591.

28. Hoogstrate $Y$, Jenster $G$, Martens-Uzunova ES. FlaiMapper: computational annotation of small ncRNA-derived fragments using RNA-seq high-throughput data. Bioinformatics. 2015;31(5):665-73. Epub 20141022. doi: 10.1093/bioinformatics/btu696. PubMed PMID: 25338717.

29. Berthelot C, Brunet F, Chalopin D, Juanchich A, Bernard M, Noel B, et al. The rainbow trout genome provides novel insights into evolution after whole-genome duplication in vertebrates. Nat Commun. 2014;5:3657. Epub 20140422. doi: 10.1038/ncomms4657. PubMed PMID: 24755649; PubMed Central PMCID: PMCPMC4071752.

30. Makino T, Mizawa M, Yoshihisa Y, Yamamoto S, Tabuchi Y, Miyai M, et al. Trichohyalin-like 1 protein plays a crucial role in proliferation and anti-apoptosis of normal human keratinocytes and squamous cell carcinoma cells. Cell Death Discov. 2020;6(1):109. Epub 20201027. doi: 10.1038/s41420020-00344-5. PubMed PMID: 33133644; PubMed Central PMCID: PMCPMC7591909.

31. Kisich KO, Howell MD, Boguniewicz M, Heizer HR, Watson NU, Leung DY. The constitutive capacity of human keratinocytes to kill Staphylococcus aureus is dependent on beta-defensin 3 . The Journal of investigative dermatology. 2007;127(10):2368-80. Epub 20070426. doi: 10.1038/sj.jid.5700861. PubMed PMID: 17460726.

32. Dong W, Rasid O, Chevalier C, Connor M, Eldridge M, Hamon MA. <em>Streptococcus pneumoniae</em> infection promotes histone H3 dephosphorylation by modulating host PP1 phosphatase. bioRxiv. 2020:2020.01.14.905968. doi: 10.1101/2020.01.14.905968.

33. Beylkin DH, Allen DL, Leinwand LA. MyoD, Myf5, and the calcineurin pathway activate the developmental myosin heavy chain genes. Dev Biol. 2006;294(2):541-53. Epub 20060403. doi: 10.1016/j.ydbio.2006.02.049. PubMed PMID: 16584724.

34. Song A, Wang Q, Goebl MG, Harrington MA. Phosphorylation of nuclear MyoD is required for its rapid degradation. Mol Cell Biol. 1998;18(9):4994-9. doi: 10.1128/MCB.18.9.4994. PubMed PMID: 9710583; PubMed Central PMCID: PMCPMC109084.

35. Robledo D, Gutiérrez AP, Barría A, Yáñez JM, Houston RD. Gene expression response to sea lice in Atlantic salmon skin: an RNA-Seq comparison between resistant and susceptible animals. bioRxiv. 2017. doi: $10.1101 / 225094$.

36. Abbas SZ, Qadir MI, Muhammad SA. Systems-level differential gene expression analysis reveals new genetic variants of oral cancer. Sci Rep. 2020;10(1):14667. Epub 20200904. doi: 10.1038/s41598-02071346-7. PubMed PMID: 32887903; PubMed Central PMCID: PMCPMC7473858.

37. Li CY, Chu JY, Yu JK, Huang XQ, Liu XJ, Shi L, et al. Regulation of alternative splicing of Bcl-x by IL-6, GM-CSF and TPA. Cell Res. 2004;14(6):473-9. doi: 10.1038/sj.cr.7290250. PubMed PMID: 15625014.

38. Jarau M, Maclnnes JI, Lumsden JS. Erythromycin and florfenicol treatment of rainbow trout Oncorhynchus mykiss (Walbaum) experimentally infected with Flavobacterium psychrophilum. J Fish Dis. 2019;42(3):325-34. Epub 20190111. doi: 10.1111/jfd.12944. PubMed PMID: 30632170. 
39. Castillo D, Christiansen RH, Dalsgaard I, Madsen L, Espejo R, Middelboe M. Comparative Genome Analysis Provides Insights into the Pathogenicity of Flavobacterium psychrophilum. PLoS One. 2016;11(4):e0152515. Epub 20160412. doi: 10.1371/journal.pone.0152515. PubMed PMID: 27071075; PubMed Central PMCID: PMCPMC4829187. 40. Sundell K, Landor L, Nicolas P, Jorgensen J, Castillo D, Middelboe M, et al. Phenotypic and Genetic Predictors of Pathogenicity and Virulence in Flavobacterium psychrophilum. Front Microbiol. 2019;10:1711. Epub 20190724. doi: 10.3389/fmicb.2019.01711. PubMed PMID: 31396199; PubMed Central PMCID: PMCPMC6668605. 1 promotes intracellular invasion by group A streptococci. Infect Immun. 2003;71(12):7119-28. Epub 2003/11/26. doi: 10.1128/iai.71.12.7119-7128.2003. PubMed PMID: 14638802; PubMed Central PMCID: PMCPMC308943.

42. Queval CJ, Song O-R, Carralot J-P, Saliou J-M, Bongiovanni A, Deloison G, et al. Mycobacterium tuberculosis Controls Phagosomal Acidification by Targeting CISH-Mediated Signaling. Cell Reports. 2017;20(13):3188-98. doi: https://doi.org/10.1016/j.celrep.2017.08.101.

43. He H, Wang Y, Guo X, Ramchandani S, Ma J, Shen MF, et al. Pot1b deletion and telomerase haploinsufficiency in mice initiate an ATR-dependent DNA damage response and elicit phenotypes resembling dyskeratosis congenita. Mol Cell Biol. 2009;29(1):229-40. Epub 2008/10/22. doi: 10.1128/mcb.01400-08. PubMed PMID: 18936156; PubMed Central PMCID: PMCPMC2612488.

44. van den Boorn JG, Dassler J, Coch C, Schlee M, Hartmann G. Exosomes as nucleic acid nanocarriers. Adv Drug Deliv Rev. 2013;65(3):331-5. Epub 20120628. doi: 10.1016/j.addr.2012.06.011. PubMed PMID: 22750807.

45. Turnbull L, Toyofuku M, Hynen AL, Kurosawa M, Pessi G, Petty NK, et al. Explosive cell lysis as a mechanism for the biogenesis of bacterial membrane vesicles and biofilms. Nat Commun. 2016;7:11220. Epub 20160414. doi: 10.1038/ncomms11220. PubMed PMID: 27075392; PubMed Central PMCID: PMCPMC4834629. aeruginosa PAO1 and their genetic transformation potential. Microbiology (Reading). 2004;150(Pt 7):2161-9. Epub 2004/07/17. doi: 10.1099/mic.0.26841-0. PubMed PMID: 15256559.

47. Kaparakis-Liaskos M, Ferrero RL. Immune modulation by bacterial outer membrane vesicles. Nat Rev Immunol. 2015;15(6):375-87. Epub 20150515. doi: 10.1038/nri3837. PubMed PMID: 25976515.

48. Anand D, Chaudhuri A. Bacterial outer membrane vesicles: New insights and applications. Mol Membr Biol. 2016;33(6-8):125-37. Epub 2016/01/01. doi: 10.1080/09687688.2017.1400602. PubMed PMID: 29189113.

49. Choi JW, Kim SC, Hong SH, Lee HJ. Secretable Small RNAs via Outer Membrane Vesicles in Periodontal Pathogens. J Dent Res. 2017;96(4):458-66. Epub 20170109. doi: 10.1177/0022034516685071. PubMed PMID: 28068479.

50. Westermann AJ, Forstner KU, Amman F, Barquist L, Chao Y, Schulte LN, et al. Dual RNA-seq unveils noncoding RNA functions in host-pathogen interactions. Nature. 2016;529(7587):496-501. Epub 20160120. doi: 10.1038/nature16547. PubMed PMID: 26789254.

51. Abendroth U, Schmidtke C, Bonas U. Small non-coding RNAs in plant-pathogenic Xanthomonas spp. RNA Biol. 2014;11(5):457-63. Epub 20140227. doi: 10.4161/rna.28240. PubMed PMID: 24667380; PubMed Central PMCID: PMCPMC4152354.

52. Soares-Silva M, Diniz FF, Gomes GN, Bahia D. The Mitogen-Activated Protein Kinase (MAPK) Pathway: Role in Immune Evasion by Trypanosomatids. Front Microbiol. 2016;7:183. Epub 20160224. doi: 10.3389/fmicb.2016.00183. PubMed PMID: 26941717; PubMed Central PMCID: PMCPMC4764696. 
53. Sjostrom AE, Sandblad L, Uhlin BE, Wai SN. Membrane vesicle-mediated release of bacterial RNA. Sci Rep. 2015;5:15329. Epub 20151020. doi: 10.1038/srep15329. PubMed PMID: 26483327; PubMed Central PMCID: PMCPMC4612299.

54. Chabelskaya S, Gaillot O, Felden B. A Staphylococcus aureus small RNA is required for bacterial virulence and regulates the expression of an immune-evasion molecule. PLoS Pathog. 2010;6(6):e1000927. Epub 20100603. doi: 10.1371/journal.ppat.1000927. PubMed PMID: 20532214; PubMed Central PMCID: PMCPMC2880579. Burkholderia cepacia cultured with subinhibitory concentrations of ceftazidime enhance proinflammatory responses. Virulence. 2020;11(1):995-1005. Epub 2020/08/18. doi: 10.1080/21505594.2020.1802193. PubMed PMID: 32799627; PubMed Central PMCID: PMCPMC7567438.

880 56. Takayama S, Reed JC, Homma S. Heat-shock proteins as regulators of apoptosis. Oncogene. 2003;22(56):9041-7. doi: 10.1038/sj.onc.1207114.

882 57. Colaco CA, Bailey CR, Walker KB, Keeble J. Heat Shock Proteins: Stimulators of Innate and Acquired Immunity. BioMed Research International. 2013;2013:461230. doi: 10.1155/2013/461230.

58. Sharma L, Feng J, Britto CJ, Dela Cruz CS. Mechanisms of Epithelial Immunity Evasion by Respiratory Bacterial Pathogens. Frontiers in Immunology. 2020;11(91). doi: 10.3389/fimmu.2020.00091. 59. Uribe-Querol E, Rosales C. Phagocytosis: Our Current Understanding of a Universal Biological Process. Frontiers in Immunology. 2020;11(1066). doi: 10.3389/fimmu.2020.01066. 60. Jo S, Kim H-R, Mun Y, Jun C-D. Transgelin-2 in immunity: Its implication in cell therapy. Journal of Leukocyte Biology. 2018;104(5):903-10. doi: https://doi.org/10.1002/JLB.MR1117-470R.

61. Mendoza JF, Cáceres JR, Santiago E, Mora LM, Sánchez L, Corona TM, et al. Evidence that G-CSF is a fibroblast growth factor that induces granulocytes to increase phagocytosis and to present a mature morphology, and that macrophages secrete 45-kd molecules with these activities as well as with G-CSFlike activity. Exp Hematol. 1990;18(8):903-10. Epub 1990/09/01. PubMed PMID: 1696904.

894

62. Ichinose M, Sawada M, Sasaki K, Oomura Y. Effect of acidic fibroblast growth factor (aFGF) on phagocytosis in mouse peritoneal macrophages. Microbiology and immunology. 1998;42(2):139-42. Epub 1998/05/08. doi: 10.1111/j.1348-0421.1998.tb02263.x. PubMed PMID: 9572047.

63. Kinchen JM, Ravichandran KS. Phagosome maturation: going through the acid test. Nature Reviews Molecular Cell Biology. 2008;9(10):781-95. doi: 10.1038/nrm2515.

64. Marshansky V, Futai M. The V-type H+-ATPase in vesicular trafficking: targeting, regulation and function. Curr Opin Cell Biol. 2008;20(4):415-26. Epub 2008/05/31. doi: 10.1016/j.ceb.2008.03.015. PubMed PMID: 18511251; PubMed Central PMCID: PMCPMC7111286.

904 65. Christoforidis S, McBride HM, Burgoyne RD, Zerial M. The Rab5 effector EEA1 is a core component of endosome docking. Nature. 1999;397(6720):621-5. doi: 10.1038/17618.

910

911

912

913

914 66. Chiou S-J, Ko H-J, Hwang C-C, Hong Y-R. The Double-Edged Sword of Beta2-Microglobulin in Antibacterial Properties and Amyloid Fibril-Mediated Cytotoxicity. International Journal of Molecular Sciences. 2021;22(12):6330. PubMed PMID: doi:10.3390/ijms22126330.

67. Cai W, Kesavan DK, Wan J, Abdelaziz MH, Su Z, Xu H. Bacterial outer membrane vesicles, a potential vaccine candidate in interactions with host cells based. Diagn Pathol. 2018;13(1):95. Epub 20181211. doi: 10.1186/s13000-018-0768-y. PubMed PMID: 30537996; PubMed Central PMCID: PMCPMC6290530.

68. Liu Q, Yi J, Liang K, Zhang X, Liu Q. Outer Membrane Vesicles Derived from Salmonella Enteritidis Protect against the Virulent Wild-Type Strain Infection in a Mouse Model. Journal of microbiology and biotechnology. 2017;27(8):1519-28. Epub 2017/05/26. doi: 10.4014/jmb.1705.05028. PubMed PMID: 28539041. 
915

916

917

918

919

920

921

922

923

924

925

926

927

928

929

930

931

932

933

934

935

936

937

938

939

940

941

942

943

944

945
69. Bottero D, Zurita ME, Gaillard ME, Bartel E, Vercellini C, Hozbor D. Membrane Vesicles Derived from Bordetella bronchiseptica: Active Constituent of a New Vaccine against Infections Caused by This Pathogen. Appl Environ Microbiol. 2018;84(4). Epub 20180131. doi: 10.1128/AEM.01877-17. PubMed PMID: 29180369; PubMed Central PMCID: PMCPMC5795063.

70. Kim D, Pertea G, Trapnell C, Pimentel H, Kelley R, Salzberg SL. TopHat2: accurate alignment of transcriptomes in the presence of insertions, deletions and gene fusions. Genome Biol. 2013;14(4):R36. Epub 20130425. doi: 10.1186/gb-2013-14-4-r36. PubMed PMID: 23618408; PubMed Central PMCID: PMCPMC4053844.

71. Li J, Ma W, Zeng P, Wang J, Geng B, Yang J, et al. LncTar: a tool for predicting the RNA targets of long noncoding RNAs. Briefings in bioinformatics. 2015;16(5):806-12. Epub 20141217. doi: 10.1093/bib/bbu048. PubMed PMID: 25524864.

72. Silverstein JT, Vallejo RL, Palti Y, Leeds TD, Rexroad CE, 3rd, Welch TJ, et al. Rainbow trout resistance to bacterial cold-water disease is moderately heritable and is not adversely correlated with growth. J Anim Sci. 2009;87(3):860-7. Epub 20081121. doi: 10.2527/jas.2008-1157. PubMed PMID: 19028851.

73. Semple SL, Bols NC, Lumsden JS, Dixon B. Understanding the pathogenesis of Flavobacterium psychrophilum using the rainbow trout monocyte/macrophage-like cell line, RTS11, as an infection model. Microb Pathog. 2020;139:103910. Epub 20191203. doi: 10.1016/j.micpath.2019.103910. PubMed PMID: 31809795.

74. Jarau M, Di Natale A, Huber PE, Maclnnes JI, Lumsden JS. Virulence of Flavobacterium psychrophilum isolates in rainbow trout Oncorhynchus mykiss (Walbaum). J Fish Dis. 2018;41(10):150514. Epub 20180803. doi: 10.1111/jfd.12861. PubMed PMID: 30074253.

75. Bertelli C, Laird MR, Williams KP, Simon Fraser University Research Computing G, Lau BY, Hoad G, et al. IslandViewer 4: expanded prediction of genomic islands for larger-scale datasets. Nucleic Acids Res. 2017;45(W1):W30-W5. Epub 2017/05/05. doi: 10.1093/nar/gkx343. PubMed PMID: 28472413; PubMed Central PMCID: PMCPMC5570257.

76. Quinlan AR, Hall IM. BEDTools: a flexible suite of utilities for comparing genomic features. Bioinformatics. 2010;26(6):841-2. Epub 20100128. doi: 10.1093/bioinformatics/btq033. PubMed PMID: $20110278 ;$ PubMed Central PMCID: PMCPMC2832824. 